\title{
Deciphering hepatocellular carcinoma through metabolomics: from biomarker discovery to therapy evaluation
}

This article was published in the following Dove Press journal:

Cancer Management and Research

\author{
Wei Guo' \\ Hor Yue Tan' \\ Ning Wang ${ }^{1,2}$ \\ Xuanbin Wang ${ }^{3,4}$ \\ Yibin Feng ${ }^{1-4}$ \\ 'School of Chinese Medicine, Li \\ Ka Shing Faculty of Medicine, The \\ University of Hong Kong, Pokfulam, \\ Hong Kong; ${ }^{2}$ Shenzhen Institute \\ of Research and Innovation, The \\ University of Hong Kong, Shenzhen, \\ China; ${ }^{3}$ Laboratory of Chinese Herbal \\ Pharmacology, Oncology Center, \\ Renmin Hospital, Hubei University of \\ Medicine, Shiyan, China; ${ }^{4}$ Hubei Key \\ Laboratory of Wudang Local Chinese \\ Medicine Research, Hubei University \\ of Medicine, Shiyan, China
}

\begin{abstract}
Hepatocellular carcinoma (HCC) is the third most common cause of death from cancer, with increasing prevalence worldwide. The mortality rate of $\mathrm{HCC}$ is similar to its incidence rate, which reflects its poor prognosis. At present, the diagnosis of HCC is still mostly dependent on invasive biopsy, imaging methods, and serum $\alpha$-fetoprotein (AFP) testing. Because of the asymptomatic nature of early HCC, biopsy and imaging methods usually detect HCC at the middle-late stages. AFP has limited sensitivity and specificity, as many other nonmalignant liver diseases can also result in a very high serum level of AFP. Therefore, better biomarkers with higher sensitivity and specificity at earlier stages are greatly needed. Since metabolic reprogramming is an essential hallmark of cancer and the liver is the metabolic hub of living systems, it is useful to investigate HCC from a metabolic perspective. As a noninvasive and nondestructive approach, metabolomics provides holistic information on dynamically metabolic responses of living systems to both endogenous and exogenous factors. Therefore, it would be conducive to apply metabolomics in investigating HCC. In this review, we summarize recent metabolomic studies on HCC cellular, animal, and clinicopathologic models with attention to metabolomics as a biomarker in cancer diagnosis. Recent applications of metabolomics with respect to therapeutic and prognostic evaluation of $\mathrm{HCC}$ are also covered, with emphasis on the potential of treatment by drugs from natural products. In the last section, the current challenges and trends of future development of metabolomics on HCC are discussed. Overall, metabolomics provides us with novel insight into the diagnosis, prognosis, and therapeutic evaluation of HCC.
\end{abstract} Keywords: hepatocellular carcinoma, metabolomics, biomarker, diagnosis and therapy

\section{Introduction}

Hepatocellular carcinoma (HCC) is the third most common cause of death from cancer, with increasing prevalence worldwide. The mortality rate of $\mathrm{HCC}$ is similar to its incidence rate, which reflects its poor prognosis. ${ }^{1} \mathrm{HCC}$ can be initiated by various factors, including genetic and environmental factors. The majority of HCC comes with a background of liver cirrhosis, primarily induced by two prominent risk factors: hepatitis $\mathrm{B}$ and $\mathrm{C}$ viruses (HBV and $\mathrm{HCV}$ ) infection. ${ }^{2}$ Chronic HBV infection consists of $52.3 \%$ and chronic HCV infection $20 \%$ of all HCC cases all over the world. ${ }^{3}$ $\mathrm{HBV}$ is the leading etiologic factor for HCC in developing regions, such as western Africa and Southeast Asia, whereas HCV contributes to the most risk in developed countries. These two potent risk factors shape the geographical variation in HCC incidence, which in the developing world is higher than that in the developed world. Also, hereditary conditions, including genetic tyrosinemia, $\alpha_{1}$-antitrypsin deficiency, and
Correspondence: Yibin Feng School of Chinese Medicine, Li Ka Shing Faculty of Medicine, The University of Hong Kong, 10 Sassoon Road,

Pokfulam, Hong Kong

Tel +85225890482

Fax +85228725476

Email yfeng@hku.hk 
hemochromatosis, can lead to HCC. An escalating amount of lifestyle-related risk factors, such as chronic alcohol abuse, tobacco smoke, betel-quid chewing, high aflatoxin intake, obesity, and diabetes, have also been linked with HCC. ${ }^{4-7}$ Invalid diagnosis and treatment of $\mathrm{HCC}$ often result in its high mortality rate, which poses a big threat to public health. Currently, the diagnosis of HCC is still mostly dependent on invasive biopsy, imaging methods, and serum $\alpha$-fetoprotein (AFP) testing. ${ }^{8}$ Because of early $\mathrm{HCC}$ asymptomatic nature, biopsy and imaging methods usually detect $\mathrm{HCC}$ at middle or late stages, for which there are no effectively therapeutic options. For the clinical diagnosis of HCC, serum AFP, a fetal serum glycoprotein, has been considered a golden biomarker. However, its sensitivity and specificity is very limited, because many other nonmalignant liver diseases, such as acute and chronic hepatitis and liver cirrhosis, can also result in a very high serum level of AFP that is similar to that detected in HCC. ${ }^{9}$ Therefore, better biomarkers are greatly needed, which would improve clinical diagnosis and therapeutic treatment of $\mathrm{HCC}$ at earlier disease stages and ultimately result in lower mortality rates. An ideal biomarker should satisfy the following conditions: target molecules with high sensitivity and specificity; markers to be detectable via a noninvasive way, such as blood and urine; and measurement techniques should be cheap, reliable, and robust across an extensive range of populations.

As the newest "-omics" science, metabolomics is the systematic study of small-molecule metabolites in living systems, which is defined as the "metabolome" (metabolites with an atomic mass $<1.5 \mathrm{kDa}$ ). ${ }^{10}$ This approach offers holistic information on dynamically metabolic responses of living systems to both endogenous and exogenous factors. The target of metabolomics is to detect and identify global small-molecule metabolic profiles of complex biological matrices. Compared with other -omics technologies (namely genomics, transcriptomics, and proteomics), where modification of substrates commonly occurs, metabolomics can provide information on metabolites that are directly produced in response to endogenous and exogenous factors. ${ }^{11}$ It provides potential biomarkers for the diagnosis and monitoring of complex diseases and response to therapeutic intervention. ${ }^{12,13}$ It has been widely applied in the domains of disease diagnosis, therapeutic monitoring, and pharmacodynamic evaluation. ${ }^{14-16}$ Cell metabolism plays an important role in cancer, and metabolic reprogramming is an essential hallmark of cancer. ${ }^{17}$ Cancer cells have been reported to own a significantly unique metabolic phenotype to support their high proliferation rates, which is highlighted by high glycolytic rates, enriched phospholipid turnover, low mitochondrial activity, and decreased bioenergetic expenditure. ${ }^{18}$ Cancer metabolism has become a "hot spot" and is gaining momentum for better mechanistic research of tumorigenesis. Therefore, it is useful to investigate cancer from a metabolic perspective.

As the metabolic hub of the human body with various metabolic functions, the liver is able to mediate the expression levels of numerous metabolites. It is well accepted that metabolic-profile variations occur prior to imaging diagnosis in HCC patients. Owing to this, HCC has become a disease model of great interest from a metabolic perspective. ${ }^{19,20}$ More specifically, metabolomics is a noninvasive and nondestructive analysis. Therefore, it would be conducive to apply metabolomics to understand the complex pathophysiology, discover new biomarkers, and evaluate new therapeutic drug targets of HCC. There has been an increasing number of metabolomic studies on HCC over the last few decades. ${ }^{21,22}$ The typical processing flow of metabolomics studies on $\mathrm{HCC}$ is shown in Figure 1. Firstly, samples for HCC are collected. There are different kinds of samples used for HCC, including in vitro HCC cells, in vivo plasma, serum, urine, feces, and liver tumor and nontumor tissue. Collected samples are preprocessed and tested by different approaches. There are two main analytical platforms used in metabolomic studies on HCC: nuclear magnetic resonance (NMR) spectroscopy and mass spectrometry (MS). The latter is usually equipped with different separation instruments, including liquid chromatography (LC), gas chromatography (GC), and capillary electrophoresis (CE). Each platform has its own strengths and weaknesses. After acquisition, the data obtained are processed and analyzed to find metabolites changed majorly by multivariate statistical analysis, such as principal-component analysis and orthogonal projection to latent structure-discriminant analysis. Lastly, according to databases and related literature, the metabolites involved and corresponding pathways are elucidated and the specific biomarkers identified.

For this review, we retrieved data from metabolomic studies on HCC in the prior 7 years from the databases PubMed and Google Scholar using the five keywords "hepatocellular carcinoma", "metabolomics", "biomarker", “diagnosis", and "therapy". First, we summarized the recent metabolomic studies on HCC cellular, animal, and clinicopathologic models with attention to its use as a biomarker in cancer diagnosis. Subsequently, recent applications of metabolomics with respect to therapeutic and prognostic evaluation of HCC were also focused upon, with emphasis on the potential of 

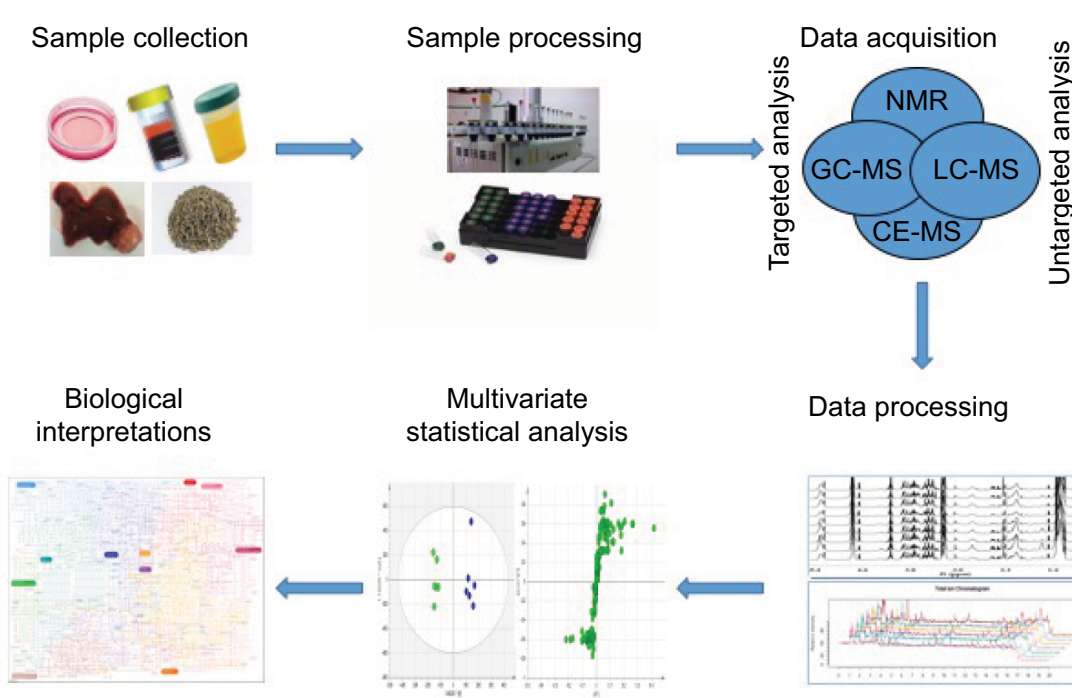

Data processing

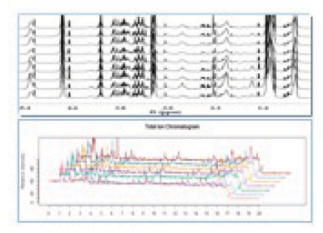

Figure I Typical processing flow of metabolomics in HCC.

Notes: Proposed standards for metabolomics on HCC are presented in this schematic view. The first step is to collect the samples. Then, the collected samples are preprocessed and tested by different approaches. After acquisition, obtained data are processed and analyzed by multivariate statistical analysis. Lastly, the underlying biological interpretations are elucidated.

Abbreviations: CE, capillary electrophoresis; GC, gas chromatography; HCC, hepatocellular carcinoma; LC, liquid chromatography; MS, mass spectrometry; NMR, nuclear magnetic resonance.

treatment by drugs from natural products. In the last section, current challenges of and trends in future development of metabolomics with regard to HCC are discussed. Overall, metabolomics provides us a novel insight into diagnosis, prognosis, and therapeutic evaluation of HCC.

\section{Metabolomics in HCC-model research}

In this section, we summarize recent metabolomic studies on HCC cellular, animal, and clinicopathologic models with attention to their use as biomarkers in cancer diagnosis. Table 1 depicts detailed information on four metabolomic studies on HCC cells and nine HCC animal models published recently, and Table 2 includes detailed information on 37 metabolomic studies of human HCC samples. Overall, these studies illustrated the majorly altered metabolites and corresponding metabolic pathways involved in stepwise hepatocarcinogenesis, suggesting that metabolomics could be a promising strategy of biomarker discovery for the early diagnosis of HCC.

\section{Cell pathology model research}

HBV infection involves intricate interactions between the virus and host cells that can induce HCC. It has been reported that there was a correlation between HBV infection and metabolic alterations in host cells. ${ }^{23,24}$ To study the underlying mechanisms of metabolic alterations caused by HBV infection, NMR-based metabolomics was used to investigate the metabolic features of HepG2.2.15 cells derived from HepG2 with stable expression and replication of HBV, which is a commonly used cell model to study HBV infection. ${ }^{25}$ The results indicated that HBV infection contributed to $\mathrm{HCC}$ by upregulation of the glutamine-fructose-6-phosphate amidotransferase 1 (GFAT1)-activated hexosamine biosynthesis and choline kinase alpha (CHKA)-activated phosphatidylcholine biosynthesis. Hepatitis B virus X protein ( $\mathrm{HBx}$ ), a multifunctional oncoprotein, was reported to be associated with HBV replication, DNA repair, cell-cycle progression, transcriptional regulation, and to play an essential role in HBV-related HCC. ${ }^{26}$ Yue et al used an NMR-based metabolomic approach to systematically investigate the effects of this specific protein on hepatocarcinogenesis. ${ }^{27}$ They found that HBx disrupted the metabolism of glucose, lipids, and amino acids, especially nucleic acids. Further investigation of the effects of HBx on nucleic acid metabolism in gene-expression profiles showed that 29 genes correlating with DNA damage and repair in HBx-expressing HepG2 cells were differentially expressed. Together, their results revealed that HBx initially caused DNA damage and then perturbed nucleic acid metabolism, which in turn blocked DNA repair and led to HCC. E4F1, a cellular target of the E1A adenoviral oncoprotein, was reported to interact with HBx. Based on combined analyses of ${ }^{1} \mathrm{H}-\mathrm{NMR}$ metabolomics and molecular biology technologies, Dai et al confirmed that E4F1 may contribute to the proliferation of HBV-infected HCC cells by neutralizing the capacity of HBx to activate a p53-dependent metabolic and growth arrest 
Table I Summary of recent metabolomics studies on HCC cellular and animal models

\begin{tabular}{|c|c|c|c|}
\hline Reference & PubMed ID & Study & Method \\
\hline 25 & 25672227 & HepG2.2.I5 and HepG2 cells & 'H-NMR \\
\hline 27 & 27075403 & HepG2 cells infected by Ad-HBx & 'H-NMR and gene-expression profiles \\
\hline 28 & 24163401 & HepG2.2.I5 and HepG2 cells & 'H-NMR \\
\hline 29 & 28112229 & HepG2 cells transfected with $\mathrm{HBc}$ & 'H-NMR and proteomics \\
\hline 30 & 24763554 & $\begin{array}{l}\text { PNPLA3 silencing and overexpression of Huh7 } \\
\text { cells }\end{array}$ & GC-MS and LC-MS \\
\hline 32 & $24 \mid 27579$ & $\begin{array}{l}\text { Primary hepatocytes from DEN-induced HCC } \\
\text { C57BL/6] }\end{array}$ & LC-MS/MS \\
\hline 33 & 22084000 & Serum from DEN-induced rat $\mathrm{HCC}$ model & LC-MS \\
\hline 34 & 26526930 & Serum from DEN-induced rat $\mathrm{HCC}$ model & CE-TOF-MS \\
\hline 35 & 27578360 & Serum from DEN-induced rat $\mathrm{HCC}$ model & LC-MS \\
\hline 36 & $208 \mid 4984$ & Serum and urine from HLM rat model & GC/TOFMS \\
\hline 37 & 20890798 & Tumor tissue from HLM rat model & 'H-NMR \\
\hline 38 & 25594851 & $\begin{array}{l}\text { Serum and liver from } \mathrm{HBx} \text { transgenic mouse } \\
\text { model }\end{array}$ & GC-MS \\
\hline 39 & 28941178 & Liver tissues from Ras-Tg mice model & $\begin{array}{l}\text { Transcriptomics and GC-TOF-MS-based } \\
\text { metabolomics }\end{array}$ \\
\hline 40 & 21763219 & Serum from nude mice bearing HepG2 cells & UHPLC/QTOF-MS \\
\hline
\end{tabular}

Abbreviations: Ad, adenovirus; AFP, $\alpha$-fetoprotein; ATSD-DN, analysis of time-series data based on dynamic networks; CE, capillary electrophoresis; CHKA, choline kinase alpha; DEN, diethylnitrosamine; ERR $\alpha$, estrogen-related receptor alpha; FFA, free fatty acid; GC, gas chromatography; GFATI, glutamine-fructose-6-phosphate amidotransferase I; $\mathrm{HBc}$, hepatitis B virus core protein; $\mathrm{HBx}$, hepatitis B virus X protein; HBV, hepatitis B virus; HCC, hepatocellular carcinoma; $\mathrm{HLM}$, HCC with lung metastasis; LC, liquid chromatography; LPC, lysophosphatidylcholine; MLX, Max-like protein X; MS, mass spectrometry; NMR, nuclear magnetic resonance; QTOF, quadrupole time of flight; TCA, tricarboxylic acid; TOF, time of flight; UHPLC, ultrahigh-performance LC.

phenotype. ${ }^{28} \mathrm{HBc}$, encoded by the HBV genome, was also reported to play an essential role in HBV-related HCC. Xie et al combined analyses of proteomics and metabolomics to investigate the function of $\mathrm{HBc}$ in $\mathrm{HBV}$-related HCC. ${ }^{29}$ They found that HBc upregulated glycolysis and amino acid metabolism, and the enriched recruitment and enrichment of Mlx by $\mathrm{HBc}$ in the nucleus were linked to glycolysis pathways. PNPLA3 is a gene regulating both acylglycerol $O$-acyltransferase and triacylglycerol lipase activities. To investigate its role in $\mathrm{HCC}$, Min et al performed GC-MS and LC-MS metabolic profiling of Huh7 cells with PNPLA3 siRNA silencing and overexpression. ${ }^{30}$ Silencing of PNPLA3 was revealed to reduce amino acid metabolism and elevate levels of myoinositol, cysteine sulfinic acid, polyunsaturated fatty acids, lysolipids, and sphingolipids. Overexpression of PNPLA3 mirrored metabolic changes in the opposite direction. Taken together, their results revealed a central role of PNPLA3 in the regulation of liver metabolism, besides its traditional role in the remodeling of triacylglycerol.

\section{Animal pathologic model research}

Different HCC animal models have been established to investigate the pathogenesis of HCC and identify specific biomarkers. Based on these models, a large number of metabolomic 


\begin{tabular}{|c|c|c|}
\hline Significantly changed metabolites or pathways & Main findings & Validation \\
\hline $\begin{array}{l}\text { HBV infection upregulated the biosynthesis of hexosamine and } \\
\text { phosphatidylcholine, induces oxidative stress, and stimulates } \\
\text { central carbon metabolism and nucleotide synthesis }\end{array}$ & $\begin{array}{l}\text { HBV-associated hepatocellular carcinoma could be attributed } \\
\text { to GFATI-activated hexosamine biosynthesis and CHKA- } \\
\text { activated phosphatidylcholine biosynthesis }\end{array}$ & Yes \\
\hline $\begin{array}{l}\text { HBx disrupted metabolism of glucose, lipids, and amino acids, } \\
\text { especially nucleic acids }\end{array}$ & $\begin{array}{l}\text { HBx initially induces DNA damage and then disrupts nucleic } \\
\text { acid metabolism, which in turn blocks DNA repair and } \\
\text { induces HCC }\end{array}$ & Yes \\
\hline $\begin{array}{l}\text { siRNA to E4FI included reduction in glutamate, glutathione, } \\
\text { acetate, and leucine, as well as increases in phosphocholine, lipid } \\
\text { glycerol, and several lipid species }\end{array}$ & $\begin{array}{l}\text { E4FI may neutralize the capacity of } \mathrm{HBx} \text { to activate a p53- } \\
\text { dependent, metabolic, and growth-arrest phenotype in liver } \\
\text { cells }\end{array}$ & No \\
\hline $\begin{array}{l}\text { Glycolysis and amino acid metabolism were significantly } \\
\text { upregulated by } \mathrm{HBc}\end{array}$ & $\begin{array}{l}\text { MLX might be recruited and enriched by } \mathrm{HBc} \text { in the nucleus } \\
\text { to regulate glycolysis pathways }\end{array}$ & No \\
\hline $\begin{array}{l}\text { Silencing of PNPLA3 declined amino acid metabolism and elevated } \\
\text { the levels of myoinositol, cysteine sulfinic acid, polyunsaturated } \\
\text { fatty acids, lysolipids, and sphingolipids, whereas overexpression } \\
\text { of PNPLA3 mirrored metabolic changes in the opposite direction }\end{array}$ & $\begin{array}{l}\text { These results suggest a critical role of PNPLA3 in the } \\
\text { modulation of liver metabolism beyond its classical } \\
\text { participation in triacylglycerol remodeling }\end{array}$ & No \\
\hline $\begin{array}{l}\text { Loss of ERR } \alpha \text { promotes hepatocyte necrosis over apoptosis in } \\
\text { response to DEN, due to a deficiency in energy production }\end{array}$ & $\begin{array}{l}\text { Loss of ERR } \alpha \text { activity promotes HCC by independent but } \\
\text { synergistic mechanisms in hepatocytes and Kupffer cells }\end{array}$ & No \\
\hline $\begin{array}{l}\text { Three metabolites - taurocholic acid, lysophosphoethanolamine } \\
\text { 16:0, and lysophosphatidylcholine 22:5 - were defined as "marker } \\
\text { metabolites" }\end{array}$ & $\begin{array}{l}\text { Three marker metabolites were effective for discrimination of } \\
\text { HCC patients, better than AFP }\end{array}$ & Yes \\
\hline $\begin{array}{l}\text { A novel biomarker pattern of creatine:betaine ratio that reflected } \\
\text { the balance of methylation was identified }\end{array}$ & $\begin{array}{l}\text { This ratio biomarker can also improve the diagnostic } \\
\text { performance of AFP }\end{array}$ & Yes \\
\hline $\begin{array}{l}\text { A ratio of LPC I8:I/FFA } 20: 5 \text { was identified as the potential } \\
\text { biomarker for HCC }\end{array}$ & $\begin{array}{l}\text { The better performance of ATSD-DN suggested its potential } \\
\text { to present time-series changes well and effectively extract } \\
\text { early-warning information }\end{array}$ & Yes \\
\hline $\begin{array}{l}\text { Glutamate metabolism and glycolysis were increased, while the } \\
\text { TCA cycle was decreased in both HCC and HLM }\end{array}$ & $\begin{array}{l}\text { Metabolism of glucuronic acid, amino acids, and nucleic acids } \\
\text { increased only in HLM }\end{array}$ & No \\
\hline $\begin{array}{l}\text { Tumor tissue from HLM showed changes in glucose, lactate, } \\
\text { choline, lipids, and some amino acids, such as glycine }\end{array}$ & $\begin{array}{l}\text { Alterations in glycolysis and the metabolism of glycine and } \\
\text { choline occur during HCC invasion and metastasis }\end{array}$ & No \\
\hline $\begin{array}{l}\text { Lipid (fatty acids, triglycerides, and cholesterol) profiles changed } \\
\text { significantly during development of } \mathrm{HBx} \text { tumorigenesis }\end{array}$ & $\begin{array}{l}\text { Metabolic syndrome plays an important role in HBV } \\
\text { tumorigenesis, and dysregulation of lipid metabolism may } \\
\text { predict disease progression to } \mathrm{HCC} \text { in chronic HBV patients }\end{array}$ & No \\
\hline $\begin{array}{l}\text { Hras I } 2 \mathrm{~V} \text { oncogene induced perturbations of glycolysis, pentose } \\
\text { phosphate pathway, TCA cycle, lipid metabolism, bile-acid } \\
\text { synthesis, and redox homeostasis }\end{array}$ & $\begin{array}{l}\text { These altered metabolic processes may play crucial roles } \\
\text { in the carcinogenesis, development, and pathological } \\
\text { characteristics of HCC }\end{array}$ & No \\
\hline $\begin{array}{l}\text { Metabolic alterations of LPCs were observed in liver injury and } \\
\text { HCC }\end{array}$ & $\begin{array}{l}\text { LPC profile in serum may be biomarker for liver injury and } \\
\text { HCC }\end{array}$ & No \\
\hline
\end{tabular}

studies have been performed. Chemicals can lead to tumorigenesis in the liver of rodents, and diethylnitrosamine (DEN) has been reported to be the most commonly used hepatocarcinogen in rodents. It has been reported that the genetic and histologic signatures of the DEN-induced HCC rodent model are similar to those of human HCC. ${ }^{31}$ Several metabolomic studies have been conducted to compare the metabolic profiles of control and HCC using DEN-induced rodents. For example, Hong et al used DEN-induced C57BL/6J model to investigate the role of estrogen-related receptor $\alpha(\mathrm{ERR} \alpha)$ in HCC. ${ }^{32}$ LC-MS/MS-based metabolomic analysis revealed that in response to $\mathrm{DEN}$, the loss of ERR $\alpha$ led to hepatocyte necrosis via apoptosis, which induced HCC through independent but synergistic mechanisms of hepatocytes and Kupffer cells. Tan et al used LC-MS-based metabolomics to identify potential biomarkers from the serum of a DEN-induced rat HCC model. ${ }^{33}$ Three metabolites - lysophosphoethanolamine 16:0, lysophosphatidylcholine (LPC) 22:5, and taurocholic acid - were identified as biomarkers and effective in discriminating HCC patients better than AFP in sensitivity and specificity. Similar samples were also analyzed via CE-time of flight (TOF)-MS-based metabolomics. ${ }^{34}$ To improve the detection of early risk of HCC, Huang et al proposed a new strategy for analysis of time-series data based on dynamic networks (ATSD-DN) using the DEN-induced rat $\mathrm{HCC}$ model. ${ }^{35}$ LC-MS-based metabolomic analysis of serum identified a ratio of lyso-phosphatidylcholine (LPC) 18:1/free fatty acid 20:5 as a biomarker of HCC. The better 
Table 2 Summary of recent metabolomic studies on HCC clinicopathologic models

\begin{tabular}{llll}
\hline Reference & PubMed ID & Study & Method \\
\hline 41 & 26130468 & Serum from II4 HCC cases and 222 matched controls & 'H-NMR \\
42 & 26399231 & $\begin{array}{l}\text { Serum from II4 primary HCC cases and 222 matched } \\
\text { controls }\end{array}$ & 'H-NMR
\end{tabular}

43

44

45

46

47

48

49

50

51

52

53

54

55

56

57

58

59
21833635

23889541

$246 I 1595$

26805550

23313056

21518826

23136190

22882828

23078175

24382646

24853826

24661807

26030804

27913395

2834097 I

22200553

25483 I 4 I
Serum from 4I HCC patients and 38 healthy controls

Serum from $29 \mathrm{HCC}$ patients and 30 age-matched healthy controls

Serum from $30 \mathrm{HCC}$ and 30 healthy volunteers

Urine from $25 \mathrm{HCC}$ patients and 12 matched healthy controls

Urine from $25 \mathrm{HCC}$ patients and 12 control subjects

Serum and urine from $82 \mathrm{HCC}$ patients, 24 benign-livertumor patients, and 7I healthy controls

Serum from 93 lung carcinomata, 28 small HCC, and 33 large HCC

Serum from 78 HCC cases and 184 cirrhotic controls

Serum from $40 \mathrm{HCC}$ patients and 49 cirrhosis patients from Egypt

Serum from $43 \mathrm{HCC}$ patients, 42 lung cirrhosis patients, and 18 healthy volunteers

Serum from 30 healthy controls, 25 cirrhosis subjects, and $22 \mathrm{HCC}$ patients

Serum from $30 \mathrm{HCC}$ patients, 27 hepatitis $\mathrm{C}$ cirrhosis disease controls and 30 healthy volunteers

Plasma from $40 \mathrm{HCC}$ cases and 49 patients with liver cirrhosis

Plasma from $63 \mathrm{HCC}$ cases and 65 cirrhotic controls

Plasma from healthy subjects $(n=20)$, patients with ESLD $(n=99)$, and patients after LTx $(n=7)$

Serum from 82 HCC, 48 lung cancer, and 90 healthy subjects
LC-MS

UHPLC/QqQ-MS

LC-MS

LC-QTOF-MS

UHPLC-QTOF-HD-MS

GC-TOF-MS and UHPLC-QTOF-MS

IH-NMR

UHPLC-QTOF-MS

UHPLC-QTOF-MS

NMR and LC-MS

CE-TOF-MS

GC-MS and UPLC-MS

GC-QMS and GC-TOF-MS

GC-MS

GC- or LC-MS

UHPLC-MS

Urine from 27 lung cirrhosis subjects, 33 HCC subjects, QTrap LC-MS and 26 healthy individuals 


Significantly changed metabolites or pathways
HCC metabolic counterpart displayed positive loading on ethanol,
glutamate, and phenylalanine
A metabolic pattern associated with HCC risk comprising
perturbations in fatty-acid oxidation and amino acid, lipid, and
carbohydrate metabolism was observed

The serum metabolite I-methyladenosine was identified as the characteristic metabolite for HCC

Lower lysophosphatidylcholine, medium-chain acylcarnitines and branched-chain amino acid, as well as enriched long-chain acylcarnitines and aromatic amino acid were observed in HCC patients

In total, 609 and I,084 ion pairs were found meeting one or more criteria for fusion

Citric acid cycle, bile-acid biosynthesis, urea-cycle metabolism, and tryptophan metabolism were significantly changed in HCC group Glycocholic acid expression was upregulated in urine samples associated with HCC

43 serum metabolites and 31 urine metabolites were identified in HCC patients involving the metabolisms of glycolysis, free fatty acids, bile acids, methionine, and urea cycle

Compared with cirrhosis, levels of glutamate, acetate, and $\mathrm{N}$-acetyl glycoproteins increased, while levels of lipids and glutamine decreased in large $\mathrm{HCC}$

Metabolites involved in sphingolipid metabolism and phospholipid catabolism upregulated in sera of HCC vs those with liver cirrhosis; downregulated metabolites included those involved in bile-acid biosynthesis (specifically cholesterol metabolism)

Significant differences between HCC and cirrhotic controls in levels of bile-acid metabolites, long-chain carnitines, and small peptides were observed

HCC induced disturbances of citrate cycle, amino acid catabolism, fatty-acid oxidation, and phospholipid metabolism and synthesis of ketone bodies, bile-acid metabolism, and sphingolipid metabolism Tryptophan, glutamine, and 2-hydroxybutyric acid were finally established

Elevated 12-hydroxyeicosatetraenoic acid (I2-HETE), I5-HETE, sphingosine, xanthine, serine, glycine, aspartate, and acylcarnitines were strongly associated with $\mathrm{HCC}$

Significant changes in levels of glutamic acid, citric acid, lactic acid, valine, isoleucine, leucine, $\alpha$-tocopherol, cholesterol, and sorbose were observed

Eleven metabolites and three clinical covariates that differentiated HCC cases from cirrhotic controls were identified

There was a significant difference in glutathione/metabolic profiles from patients with ESLD vs healthy subjects and patients after LTx

There were significant disturbances of key metabolic pathways in HCC patients, such as organic acids, phospholipids, fatty acids, bile acids, and gut-flora metabolism

Hydantoin-5-propionic acid and butyrylcarnitine (carnitine C 4:0)

were identified as combinational markers to distinguish $\mathrm{HCC}$ from lung cirrhosis
Main findings

Validation

This study devised a way to bridge lifestyle variables to

No

HCC risk through NMR metabolomic data

Results showed clear metabolic alterations from early stages of HCC development, with application for better etiologic understanding, prevention, and early detection of $\mathrm{HCC}$

A combination of I-methyladenosine and AFP exhibited significantly improved sensitivity

This work provides an approach to acquire multiplereaction-monitoring ion pairs from real samples and a foundation to achieve pseudotargeted metabolomic analysis

The developed method can be an effective tool to process high-resolution mass spectrometry data in "omics" studies Five marker metabolites were effective for diagnosis of human HCC

The network generation clearly enhances the interpretation and understanding of mechanisms for glycocholic acid

The identified biomarkers differentiated HCC patients

No with AFP levels lower than $20 \mathrm{ng} / \mathrm{mL}$ from healthy controls with $100 \%$ accuracy

Serum NMR-based metabolomics identified metabolic fingerprints that could be specific to large HCC in cirrhotic livers

Metabolic profiling is a promising tool to identify candidate metabolic biomarkers for early detection of HCC cases in high-risk population of cirrhotic patients

This was the first MS-based metabolic biomarkerdiscovery study on Egyptian subjects

These potential biomarkers appeared to have diagnostic and/or prognostic values for HCC, which deserve to be further investigated

Serum-biomarker model enabled the discrimination of small HCC from precancer cirrhosis with an AUC of 0.976

Aberrant amino acid biosynthesis, cell-turnover regulation, reactive oxygen-species neutralization, and eicosanoid pathways may be hallmarks of HCC Findings indicated upregulation of metabolites involved in branched-chain amino acid metabolism

This study demonstrated the combination of metabolites and clinical covariates as an effective approach for early detection of HCC in patients with liver cirrhosis Glutathione species and metabolic prints defined liverdisease severity and may serve as surrogates for the detection of HCC in patients with established cirrhosis glycochenodeoxycholic acid was suggested to be an important indicator for HCC diagnosis and disease prognosis

The established pseudotargeted method is a complementary one of targeted and nontargeted methods for metabolomic study 
Table 2 (Continued)

\begin{tabular}{|c|c|c|c|}
\hline Reference & PubMed ID & Study & Method \\
\hline 60 & 21275434 & $\begin{array}{l}\text { Urine from } 20 \text { healthy controls, } 20 \text { lung cirrhosis and } 18 \\
\text { HCC patients }\end{array}$ & 'H-NMR \\
\hline 61 & 27862090 & $\begin{array}{l}\text { Urine from } 40 \text { lung cirrhosis, } 55 \mathrm{HCC} \text {, and } 45 \text { healthy } \\
\text { male subjects }\end{array}$ & GC-MS \\
\hline 62 & 24817358 & $\begin{array}{l}\text { Urine from } 2 \text { I healthy controls, } 2 \text { I lung cirrhosis, and } 28 \\
\text { HCC patients }\end{array}$ & LC-MS \\
\hline 63 & 21458633 & $\begin{array}{l}\text { Feces from } 23 \text { healthy, } 22 \text { lung cirrhosis, and } 23 \mathrm{HCC} \\
\text { individuals }\end{array}$ & UHPLC/QTOF-MS \\
\hline 64 & 21900402 & $\begin{array}{l}\text { Plasma from } 20 \text { HCC, } 7 \text { lung cirrhosis, } 22 \text { acute myeloid } \\
\text { leukemia patients, and } 6 \text { healthy volunteers }\end{array}$ & $\begin{array}{l}\text { UHPLC-ESI-QTOF-MS, UHPLC-ESI-TQ- } \\
\text { MS, and GC-MS }\end{array}$ \\
\hline 65 & $2294684 I$ & $\begin{array}{l}\text { Serum from } 28 \text { lung cirrhosis ( } 22 \text { with HBV infection and } \\
6 \text { with HCV infection), } 69 \mathrm{HCC} \text { ( } 38 \text { with HBV infection } \\
\text { and } 31 \text { with } \mathrm{HCV} \text { infection), and } 31 \text { healthy volunteers }\end{array}$ & UHPLC-QTOF-MS \\
\hline 66 & 24707821 & $\begin{array}{l}\text { Serum from } 30 \text { lung cirrhosis ( } 22 \text { infected with } \mathrm{HBV} \text { and } \\
8 \text { with } \mathrm{HCV}), 70 \mathrm{HCC} \text { ( } 39 \text { with } \mathrm{HBV} \text { infection and } 3 \mathrm{I} \\
\text { with } \mathrm{HCV} \text { infection), and } 31 \text { healthy volunteers }\end{array}$ & LC-MS \\
\hline 67 & 24666728 & Serum from $37 \mathrm{HCC}$ patients and $2 \mathrm{I} \mathrm{HCV}$ patients & HPLC-MS \\
\hline 68 & 24957758 & $\begin{array}{l}\text { Serum samples from patients with HCC }(n=40) \text { and HCV } \\
(n=22)\end{array}$ & 'H-NMR \\
\hline 69 & 23856972 & $\begin{array}{l}\text { Serum from } 30 \mathrm{HCC} \text { patients with underlying } \mathrm{HCV} \text { and } \\
\text { cirrhosis and } 22 \mathrm{HCV} \text { patients with cirrhosis but without } \\
\mathrm{HCC}\end{array}$ & LC-MS/MS \\
\hline 70 & 26658617 & $\begin{array}{l}\text { Serum from } 49 \mathrm{HBV} \text { patients, } 52 \text { lung cirrhosis patients, } \\
39 \mathrm{HCC} \text { patients, and } 6 \mathrm{I} \text { healthy subjects }\end{array}$ & GC-TOF-MS \\
\hline 71 & 28969038 & $\begin{array}{l}\text { Serum from } 49 \mathrm{HBV} \text {-cirrhosis patients, } 5 \mathrm{I} \mathrm{HCC} \text { patients } \\
\text { and } 39 \text { healthy subjects }\end{array}$ & GC-MS and LC-MS \\
\hline 72 & 24939728 & $\begin{array}{l}\text { Serum from } 30 \text { healthy controls, } 30 \text { chronic hepatitis B } \\
(\mathrm{CHB}) \text { patients, } 30 \text { lung cirrhosis patients, and } 30 \mathrm{HCC} \\
\text { patients }\end{array}$ & UHPLC-MS \\
\hline 73 & 22349331 & $\begin{array}{l}\text { Serum from } 30 \text { healthy volunteers and } 90 \text { patients with } \\
\text { liver diseases ( } 30 \mathrm{CHB}, 30 \text { lung cirrhosis, and } 30 \mathrm{HCC})\end{array}$ & LC-MS \\
\hline 74 & 21334394 & $\begin{array}{l}\text { Serum from } 248 \text { patients with } 9 \text { types of liver disease and } \\
\text { healthy controls }\end{array}$ & CE-MS \\
\hline 75 & 23824744 & $\begin{array}{l}50 \text { pairs of liver cancer samples and matched normal } \\
\text { tissues }\end{array}$ & LC-MS \\
\hline
\end{tabular}


Significantly changed metabolites or pathways

Discriminatory metabolites included glycine, trimethylamine- $\mathrm{N}$-oxide,

hippurate, citrate, creatinine, creatine, and carnitine

13 marker metabolites (glycine, serine, threonine, proline, urea, phosphate, pyrimidine, arabinose, xylitol, hippuric acid, citric acid, xylonic acid, and glycerol) responsible for the separation of HCC group from healthy subjects

Severe disorders of steroid-hormone network and holistically decreased urinary steroid hormone pattern in cirrhotic and early HCC patients were observed

Compared with healthy controls, lysophosphatidylcholines significantly increased, while bile acids and bile pigments significantly decreased in lung cirrhosis and HCC

$\mathrm{HCC}$ associated with increased plasma levels of glycodeoxycholate, deoxycholate 3-sulfate, and bilirubin

Serum endocannabinoids anandamide (AEA) and palmitoylethanolamide (PEA) significantly elevated in HCC with HCV compared to corresponding chronic liver diseases Multi-TGDR global model selected 45 metabolites with no misclassification and multi-TGDR local selected 48 metabolites with no misclassification

The levels of cholylglycine, xanthine, uric acid, dioleoylphosphatidylcholine, arachidonyl lysolecithin, 3-hydroxycapric acid, and d-leucic acid were found to alter in HCC

Three metabolites (choline, valine, and creatinine) significantly altered in $\mathrm{HCC}$

A number of perturbed metabolic pathways, including amino acid, purine, and nucleotide metabolism, were identified

$\beta$-glutamate and asparagine for HCC vs lung cirrhosis, palmitic acid for lung cirrhosis vs HBV, 5-methoxytryptamine, malic acid, and phenylalanine for HBV vs NC were selected as potential liverdisease-specific biomarkers

Malate, citrate, succinate, lysine, carnitine, proline, ornithine, serine, phenylalanine, tyrosine, arachidonic acid, arabinose, galactose, uric acid, and several prostaglandins and leukotrienes implicated in pathological processes in HBV-cirrhosis and HCC

Based on the 27 selected feature pairs, HCC and chronic liver diseases were accurately distinguished using principal-component analysis

Long-chain acylcarnitines accumulated, whereas free carnitine and medium- and short-chain acylcarnitines decreased with the severity of nonmalignant liver diseases

Measurement of $\gamma$-glutamyl dipeptide levels distinguished among different liver diseases

Glycolysis, gluconeogenesis, and $\beta$-oxidation increased, while TCA cycle and $\Delta \mathrm{I} 2$ desaturase decreased in $\mathrm{HCC}$ tumors
Main findings

Validation

This was the first study to identify similarly altered urine metabolic profiles of HCC in two etiologically and ethnically distinct populations

Metabolic profile using GC-MS established optimized diagnostic model to discriminate between HCC patients and healthy subjects

A panel of two urinary steroid hormones (epitestosterone and allotetrahydrocortisol) displayed excellent diagnostic capability distinguishing early HCC from cirrhosis Results demonstrate the potential of UHPLC-MS as an efficient and convenient method for the early diagnosis of cirrhosis and HCC

This investigation illustrates the power of new discovery technologies represented by the UHPLC-ESI-QTOF-

MS platform combined with the targeted, quantitative platforms of UHPLC-ESI-TQ-MS and GC-MS for conducting metabolomic investigations

AEA, PEA, or their combination are potential biomarkers to distinguish the $\mathrm{HCC}$ from cirrhosis infected with $\mathrm{HCV}$

Multi-TGDR local is recommended because it has similar predictive performance and requires the same computing time as multi-TGDR global, but may provide class-specific inference

The use of biological significance as a selection process prior to PLS-DA modeling may offer improved probabilities for translation of newly discovered biomarkers to clinical application

Metabolite profiling could provide an alternative approach for $\mathrm{HCC}$ screening in $\mathrm{HCV}$ patients

These results provide a promising methodology to distinguish cirrhotic HCV patients who are at high risk of developing $\mathrm{HCC}$ from those who have already progressed to $\mathrm{HCC}$

All metabolic perturbations in these liver diseases associated with pathways for energy metabolism, macromolecular synthesis, and maintaining redox balance

These identified biomarkers possessed strong potential to distinguish and diagnose $\mathrm{HCC}$ from healthy controls and HBV-cirrhosis patients

Certain profound metabolic disturbances related to liverdisease development were revealed by the feature pairs

General changing extent was smaller in HCC than in lung cirrhosis, possibly due to the special energy-consumption mechanism of tumor cells

$\gamma$-Glutamyl dipeptides are novel biomarkers for liver diseases

Combination biomarker of betaine and propionylcarnitine useful for diagnosis of HCC. with a supplementary role for AFP
No

No

No

No

No

No 
Table 2 (Continued)

\begin{tabular}{llll}
\hline Reference & PubMed ID & Study & Method \\
\hline 76 & 23801834 & Central tumor tissue, adjacent tissue, and distant tissue & UHPLC-MS \\
& from I0 HBV-related HCC patients
\end{tabular}

77

78

79

80
23376425

23463346

26538415

24497316
Paired tumor and nontumor tissues from 30 patients with HCC

3I pairs of $\mathrm{HCC}$ tumors and corresponding nontumor liver tissue

$10 \mathrm{ICC}$ and $6 \mathrm{HCC}$ samples and their respective surrounding nontumor tissues

Human HCC, lung cirrhosis, and nontumor liver tissue
Gene expression, LC-MS, and GC-MS

Transcriptomics and GC-MS-based metabolomics

Transcriptomics and CE-TOF-MS-based metabolomics

Transcriptomics, NMR, and LC-MS/MSbased metabolomics

Abbreviations: AFP, $\alpha$-fetoprotein; CE, capillary electrophoresis; ESI, electrospray ionization; ESLD, end-stage liver disease; HBV, hepatitis B virus; HCV, hepatitis C virus; GC, gas chromatography; HCC, hepatocellular carcinoma; HD, high-definition; ICC, intrahepatic cholangiocarcinoma; LC, liquid chromatography; LTx, liver transplantation; MS, mass spectrometry; NC, normal controls; NMR, nuclear magnetic resonance; PLS-DA, projection to latent structure-discriminant analysis; QqQ-MS, triple-quadrupole mass spectrometry; QTOF, quadrupole time of flight; SCD, stearoyl-CoA-desaturase; TCA, tricarboxylic acid; TGDR, threshold gradient descent regularization; TOF, time of flight; TQ, triple quadrupole; UHPLC, ultrahigh-performance LC.

performance of ATSD-DN suggested its potential to represent time-series changes well and effectively extract early-warning information. Also, the DEN-induced rat HCC model can develop lung metastatic nodules and has also been used to investigate the metastasis of $\mathrm{HCC}$, which contributes to the poor prognosis and high mortality rate of HCC. Li et al used a DEN-induced rat HCC with lung metastasis (HLM) model to study serum and urine metabolic profiles via GC/TOF-MS metabolomics. ${ }^{36}$ They found that glutamate metabolism and glycolysis were increased and the tricarboxylic acid (TCA) cycle decreased in both HCC and HLM. Especially, the metabolism of glucuronic acid, amino acids, and nucleic acids were increased only in HLM, which revealed potential biomarkers for HCC invasion and metastasis. Wang et al also used this model to study tumor-tissue metabolic profiles via ${ }^{1} \mathrm{H}-\mathrm{NMR}$ metabolomics. ${ }^{37}$

The transgenic mouse model is also used to investigate the pathogenesis of HCC. Teng et al used an HBx transgenic mouse model to study HBV-associated HCC pathogenesis. ${ }^{38}$ GC-MS-based metabolic profiles of serum and liver revealed that lipid (fatty acids, triglycerides, and cholesterol) profiles changed significantly during the development of HBx tumorigenesis, supporting the view that metabolic syndrome is essential in HBV tumorigenesis. Fan et al used a Ras-Tg mice model to study Hras $^{12 \mathrm{~V}}$ oncogene-associated HCC pathogenesis. ${ }^{39}$ The combined analysis of transcriptomics and GCTOF-MS-based metabolomics revealed that ras $^{12 \mathrm{~V}}$ induced perturbations of glycolysis, the pentose phosphate pathway, the TCA cycle, lipid metabolism, bile-acid synthesis, and redox homeostasis. These altered metabolic pathways may be essential to HCC. In addition, an HCC-xenograft model has also been used. Alcohol abuse is one of the main causes of liver injury that can promote HCC. To investigate the underlying pathogenesis of HCC from liver injury, an HCCxenograft model was built by subcutaneously inoculating HepG2 cells into nude mice. ${ }^{40}$ Ultrahigh-performance LC (UHPLC)/quadrupole TOF (QTOF)-MS-based metabolic profiles of serum revealed metabolic alterations of LPCs in liver injury and $\mathrm{HCC}$, suggesting that the serum profile of LPC may be a biomarker for liver injury and HCC.

\section{Clinicopathologic model research}

Apart from research on $\mathrm{HCC}$ cellular and animal pathologic models, there have been a larger number of metabolomic studies on HCC clinicopathologic models. Since blood, urine, and feces can be easily obtained from humans without any invasion, they are commonly used in human metabolomic studies. A number of metabolomic studies of blood and urine to distinguish $\mathrm{HCC}$ from healthy controls have been reported. Assi et al performed ${ }^{1} \mathrm{H}-\mathrm{NMR}$-based metabolomics on prediagnostic serum from 114 first-incident, primary HCC cases and 222 matched controls identified from among the participants of a large European prospective cohort. ${ }^{41,42}$ They found that the changed metabolites in the HCC group were positively related to HCC risk. LC-MSbased metabolomic studies have also been done. ${ }^{43-47}$ Chen et al performed LC-MS-based metabolomics on serum from $41 \mathrm{HCC}$ patients and 38 healthy controls. ${ }^{43}$ They identified 


\begin{tabular}{lll}
\hline Significantly changed metabolites or pathways & Main findings & Validation \\
\hline $\begin{array}{l}\text { I4 metabolites were identified as characteristic metabolites that } \\
\text { showed significant differences in levels between central tumor tissue } \\
\text { and distant tumor tissue }\end{array}$ & $\begin{array}{l}\text { Characteristic metabolites and metabolic pathways highly } \\
\text { related to HCC pathogenesis and progression identified } \\
\text { through metabolic-profiling analysis of HCC-tissue } \\
\text { homogenates } \\
\text { A lipogenic network that involves SCD and palmitate } \\
\text { signaling was identified to be associated with HCC } \\
\text { ponounsaturated palmitic acid, a product of SCD, elevated in }\end{array}$ & $\begin{array}{l}\text { Tissue metabolomics yielded precise biochemical } \\
\text { information regarding HCC-tumor metabolic remodeling } \\
\text { from mitochondrial oxidation to aerobic glycolysis }\end{array}$ \\
$\begin{array}{l}\text { Levels of glucose, malate, myoinositol, alanine, linoleic acid, and } \\
\text { glycerol 2- and -3-phosphate decreased in HCC }\end{array}$ & $\begin{array}{l}\text { ICC and HCC have different carcinogenic mechanism, so } \\
\text { knowing the specific profile of genes and compounds can }\end{array}$ \\
$\begin{array}{l}\text { There were I4 compounds, 62 mRNAs and I7 miRNAs with two } \\
\text { distinct patterns: tumor and nontumor, and ICC and non-ICC }\end{array}$ & $\begin{array}{l}\text { be useful in diagnosing ICC } \\
\text { Alanine, succinate, lactate, glycerophosphoethanolamine, } \\
\text { aspartate metabolism, branched-chain amino acid metabolism, and }\end{array}$ \\
TCA metabolism differentiable in HCC compared to nontumor liver & Norganic phosphate were potential biomarkers
\end{tabular}

the serum 1-methyladenosine as a characteristic metabolite for HCC, and a combination of 1-methyladenosine and AFP showed significantly improved sensitivity. Chen et al conducted UHPLC/triple - quadrupole MS-based metabolomics on serum from 29 HCC patients and 30 age-matched healthy controls. ${ }^{44}$ Lower LPC, medium-chain acylcarnitines, branched-chain amino acids and enriched long-chain acylcarnitines, and aromatic amino acids were observed in HCC patients. Liang et al conducted LC-QTOF-MS-based metabolomics on urine from $25 \mathrm{HCC}$ patients and 12 matched healthy controls. ${ }^{46}$ The citric acid cycle, bile-acid biosynthesis, urea-cycle metabolism, and tryptophan metabolism were significantly changed in the HCC group. In addition, a study on serum and urine metabolic profiles from $82 \mathrm{HCC}$ patients, 24 benign liver tumor patients, and 71 healthy controls was conducted by a combination of GC-TOF-MS and UHPLCQTOF-MS metabolomics: ${ }^{48} 43$ serum metabolites and 31 urine metabolites were identified in HCC patients involving the metabolism of glycolysis, free fatty acids, bile acids, methionine, and the urea cycle. The identified biomarkers differentiated HCC patients with AFP levels lower than 20 $\mathrm{ng} / \mathrm{mL}$ from healthy controls with $100 \%$ accuracy.

The early and accurate discrimination of $\mathrm{HCC}$ from other high-risk liver diseases, such as $\mathrm{HBV}, \mathrm{HCV}$, and liver cirrhosis, can improve the prognosis of $\mathrm{HCC}$ patients. As the majority of HCC comes with a background of liver cirrhosis, many studies have attempted to discriminate the serum, plasma, urine, or feces metabolic profiles of HCC from liver cirrhosis. ${ }^{49-64}$ For example, Nahon et al performed ${ }^{1} \mathrm{H}-\mathrm{NMR}$-based metabolomics on serum from 93 liver cirrhosis, 28 small HCC, and
33 large HCC samples. ${ }^{49}$ Compared with cirrhosis, levels of glutamate, acetate, and $N$-acetyl glycoproteins were increased and levels of lipids and glutamine largely decreased in HCC. A combination of NMR and LC-MS-based metabolomics was applied to study serum metabolic profiles from $43 \mathrm{HCC}$ patients, 42 liver cirrhosis patients, and 18 healthy volunteers. ${ }^{52}$ The results revealed that HCC induced disturbances of the citrate cycle, amino acid catabolism, fatty-acid oxidation, phospholipid metabolism, synthesis of ketone bodies, bile-acid metabolism, and sphingolipid metabolism. Sanabria et al conducted GC/LC-MS-based metabolomic analyses on plasma from healthy subjects $(n=20)$, patients with end-stage liver disease $(n=99)$, and patients after liver transplantation $(n=7)$. The results showed glutathione species may define liver disease severity and serve as surrogates for the early detection of $\mathrm{HCC}$ in patients with established cirrhosis. ${ }^{57}$ Shao et al conducted QTrap LC-MS-based metabolomics on urine from 27 liver cirrhosis subjects, $33 \mathrm{HCC}$ subjects, and 26 healthy individuals,${ }^{59}$ identifying hydantoin-5-propionic acid and butyrylcarnitine (carnitine $\mathrm{C} 4: 0$ ) as combinational biomarkers to distinguish $\mathrm{HCC}$ from liver cirrhosis. Cao et al performed UHPLC/QTOF-MS-based metabolomics on feces from 23 healthy individuals, 22 with liver cirrhosis, and 23 with HCC. ${ }^{63}$ Compared with healthy controls, LPC was significantly increased and bile acids and bile pigments significantly decreased in liver cirrhosis and $\mathrm{HCC}$, which were identified as potential biomarkers. Since $\mathrm{HBV} / \mathrm{HCV}$ infection is the prominent inducer of $\mathrm{HCC}$, there have been several metabolomic investigations on the metabolic differences between hepatitis patients and HCC patients. ${ }^{65-74}$ Bowers et al 
performed HPLC-MS-based metabolomics on serum from 37 HCC patients and $21 \mathrm{HCV}$ patients.$^{67}$ Levels of cholylglycine, xanthine, uric acid, dioleoylphosphatidylcholine, arachidonyl lysolecithin, 3-hydroxycapric acid, and D-leucic acid were found to be altered in $\mathrm{HCC}$, and may be potential biomarkers to distinguish HCC from HCV. Wei et al distinguished HCC from HCV by three metabolites (choline, valine, and creatinine) with ${ }^{1} \mathrm{H}-\mathrm{NMR}$-based serum metabolomics. ${ }^{68}$ Gao et al performed GC-TOF-MS-based metabolomics on serum from $49 \mathrm{HBV}$ patients, 52 liver cirrhosis patients, 39 HCC patients, and 61 healthy subjects (normal controls $[\mathrm{NC}]) .{ }^{70} \beta$-Glutamate and asparagine for HCC versus liver cirrhosis, palmitic acid for liver cirrhosis versus HBV, and 5-methoxytryptamine, malic acid, and phenylalanine for $\mathrm{HBV}$ versus NC were selected as potential liver disease-specific biomarkers. Gong et al used a combination of untargeted metabolomics and targeted eicosanoid analysis on serum from $49 \mathrm{HBV}$-cirrhosis patients, $51 \mathrm{HCC}$ patients, and $39 \mathrm{NC}$. The biomarkers identified in this study showed high potential to differentiate $\mathrm{HCC}$ from $\mathrm{NC}$ and $\mathrm{HBV}$-cirrhosis patients. ${ }^{71}$

Besides the commonly used blood and urine samples in clinical metabolomic studies, there have also been metabolomic studies based on human tissue. ${ }^{75-80}$ The liver is the most essential metabolic organ, and liver extracts can provide complex metabolic information. Additionally, integration of metabolomics with other -omics technologies can be achieved when the sample used is tissue. For example, Budhu et al performed an integration of gene-expression technology, LC-MS, and GC-MS-based metabolomics on paired tumor and nontumor tissues from $30 \mathrm{HCC}$ patients: ${ }^{77} 169$ genes and 28 metabolites were identified to be related to HCC. Specifically, monounsaturated palmitic acid, a product of stearoyl-CoAdesaturase, was elevated in aggressive HCC and confirmed to increase the invasion and migration of HCC cells in vitro. Beyoğlu et al conducted an integration of transcriptomics and GC-MS-based metabolomics on 31 pairs of HCC tumors and corresponding nontumor liver tissues. ${ }^{78}$ The metabolomic results showed that levels of glucose, malate, myoinositol, alanine, linoleic acid, and glycerol 2- and 3-phosphate were decreased in HCC. Transcriptomics classified HCC in G1-G6 subgroups, and suggested that the high serum level of AFP in G1 was connected to the overexpression of lipid catabolic enzymes. Altogether, HCC highlighted metabolic remodeling from mitochondrial oxidation to aerobic glycolysis. In addition, the combination of metabolomics with other -omics technologies could also be used to study the differences between $\mathrm{HCC}$ and intrahepatic cholangiocarcinoma. ${ }^{79}$

\section{Metabolomics in treatment evaluation}

Besides metabolomic studies on $\mathrm{HCC}$, recent applications of metabolomics with respect to therapeutic and prognostic evaluation of HCC have also focused on the potential of treatment by drugs from natural products (Table 3). Metabolomics provides comprehensive metabolic profiles of living systems in response to drug treatment or surgery, which further helps us to better understand the underlying mechanism of antiHCC agents and predict the risk of tumor recurrence.

\section{Metabolomics in anti-HCC-agent research}

Chemotherapy remains one of the main approaches for the treatment of HCC. Specifically, metabolomics can be used to study the effects of cotreatment of currently approved drugs. NMR-based metabolomics was applied to investigate the effects of belinostat and bortezomib cotreatment on HCC. ${ }^{81}$ The cotreatment increased amino acids and induced oxidative stress, displaying synergistic antiproliferative properties. A similar synergistic anti-HCC effect was also found in sorafenib-everolimus combination therapy using 2-D high-resolution magic-angle spinning ${ }^{1} \mathrm{H}-\mathrm{NMR}$ metabolomics. ${ }^{82}$ So far, most chemotherapeutic drugs have not been effective for $\mathrm{HCC}$, due to drug resistance and serious side effects. ${ }^{83}$ Therefore, it is urgently necessary to develop new anti-HCC agents. Due to their multiple biological activities and low toxicity, natural products obtained from Chinese medicine have been explored to improve HCC prognosis and survival rate. The in vitro application of metabolomics for efficacy evaluation of anti-HCC agents from natural products has been shown to be predictive of treatment efficacy. Geranylgeranic acid (GGA), an acyclic diterpenoid, is found in medicinal herbs, such as turmeric. It has been reported to induce the death of HCC Huh7 cells. Iwao et al used UHPLC/TOF-MS-based metabolomics to study the underlying mechanism of its anti-HCC effect. ${ }^{84}$ Their results showed that GGA could increase fructose 6-phosphate and spermine while decreasing fructose 1,6-diphosphate and spermidine, suggesting that GGA may shift Huh7 cells from aerobic glycolysis to mitochondrial respiration via upregulating TIGAR and SCO2 protein levels. Dehydroepiandrosterone (DHEA), a steroid secreted by the brain, gastrointestinal tract, adrenal cortex and gonads, is reported to have antiproliferative properties. A rapid-resolution LC-TOF-MS metabolomic approach revealed that DHEA treatment caused changes in metabolism of glutathione, lipids, $S$-adenosylmethionine, 
and lysine, suggesting that the mitochondrial dysfunction caused by decreased $S$-adenosylmethionine production and cardiolipin depletion underlay the antiproliferative effect of DHEA. ${ }^{85}$ Ikuta et al used GC-MS metabolomics to evaluate the effect of R- $\alpha$-lipoic acid (RLA) on rat HCC H4IIEC 3 cells. ${ }^{86}$ The results showed RLA treatment inhibited gluconeogenesis via suppressing Thr-Gly-Ser pathways, glycolysis, and lactic acid production, providing the mechanism on how RLA induces apoptosis in HCC cells. Acyclic retinoid (ACR), a synthetic retinoid, was shown to prevent HCC but not normal hepatic cells. Qin et al applied NMR and CE-TOF-MS-based metabolomics to investigate the molecular basis of its selective anti-HCC effect. ${ }^{87}$ It was found that ACR treatment suppressed adenosine triphosphate, suggesting that ACR played a selective anti-HCC effect via suppression of the enhanced energy metabolism of HCC but not normal hepatic cells. Noguchi et al used GC-MS-based metabolomics to investigate the effects of cotreatment of palmitate (PA) and oleate (OA) on HCC. ${ }^{88}$ They found that metabolite levels for TCA-cycle intermediates, pentose phosphate-pathway intermediates, and energystorage metabolites changed between PA treatment alone and PA-OA cotreatment. This demonstrated that abnormal pentose phosphate-pathway fluxes and increased adenosine levels might contribute to differences between PA treatment alone and PA-OA cotreatment.

Metabolomics has also been applied to evaluate the efficacy of anti-HCC natural products in vivo. For example, 6,7-dimethoxy-1,2,3,4-tetrahydro-isoquinoline-3-carboxylic acid is an isoquinoline alkaloid extracted from Mucuna pruriens seeds and reported to have antiproliferative action. Kumar et al used ${ }^{1} \mathrm{H}-\mathrm{NMR}$-based metabolomics of serum from $\mathrm{HCC}$ rats to investigate the underlying mechanism. ${ }^{89}$ The results showed that 6,7-dimethoxy-1,2,3,4-tetrahydroisoquinoline-3-carboxylic acid treatment modulated fatty acids, low-density lipoproteins, acetoacetate, choline, lysine, leucine, isoleucine, tyrosine, pyruvate, and creatine to normal levels, and amelioration of these markers may be linked to repair of inflammation damage, improvement in energy metabolism, and reconstruction of cell-membrane injury. Li et al applied UHPLC-QTOF-MS-based metabolomics of urine samples from mice bearing $\mathrm{H} 22$ cells to investigate the anti-HCC effect of hispidulin, which is found widely in Chinese herbs. ${ }^{90}$ Physapubenolide is a withanolide extracted from Physalis pubescens. Ma et al applied GC-MS-based metabolomics to explore its anti-HCC mechanism. ${ }^{91}$ They found that physapubenolide significantly decreased lactate production and suppressed glycolysis via the Akt-p53 pathway. Similar anti-HCC effects of ACR were also explored via CE-TOF-MS- and LC-TOF-MS-based metabolomics of liver tissue samples from the DEN-induced HCC mouse model.$^{92}$ Metabolomics can also be applied to investigate the anti-HCC effect of traditional Chinese medicine formulae. For example, shuihonghuazi formula (SHHZF) is a traditional Chinese medicine made from four herbs, namely Semen Coicis, Fructus Polygoni Orientalis, Imperatae Rhizoma, and Ophicalcitum. SHHZF has been applied to treat HCC clinically, and its underlying mechanism was investigated in liver tissue from a DEN-induced HCC rat model via HPLC/ electrospray ionization-TOF-MS-based metabolomics. ${ }^{93}$ It was observed that SHHZF inhibited abnormal fatty-acid and bile-acid metabolism, and its anti-HCC property was achieved via regulating the activities of lysophospholipase D, MTHFR, and PEMT.

\section{Metabolomics in prognostic evaluation}

Surgery is the common curative approach for HCC patients, yet predicting the risk of tumor recurrence after surgery is difficult. Prognosis estimation in HCC patients is important, which can offer essential information on diagnosis and thus indicate treatment. Metabolomics is also applied to evaluate the prognosis of HCC. For example, Zhou et al used LC-MSbased metabolomics of plasma to predict early postoperative recurrence among HCC patients. ${ }^{94}$ They collected plasma samples from 22 early-recurrent and 18 late-recurrent $\mathrm{HCC}$ patients. Compared with the late-recurrent HCC group, fattyacid and bile-acid steroids in the early-recurrent HCC group were found to change greatly. Specifically, decreased levels of linolenic acid, docosahexaenoic acid, and polyunsaturated eicosapentaenoic acid were the specific biomarkers for early recurrence. Ye et al utilized GC-TOF-MS-based metabolomics to investigate the complex physiopathological regulations of HCC after surgical resection. ${ }^{95}$ They collected urinary samples from 19 pairs of matched preoperative and postoperative HCC patients and 20 healthy volunteers. Their results showed that metabolism of the TCA cycle, amino acids, nucleosides, and gut flora significantly changed after surgical resection, and five metabolites (acotinic acid, phenylalanine, ethanolamine, ribose, and lactic acid) were identified as biomarkers to predict early recurrence. Goossens et al applied ${ }^{1} \mathrm{H}-\mathrm{NMR}$-based metabolomics to investigate the risk of tumor recurrence in HCC patients. ${ }^{96}$ They collected serum samples from 120 HCC patients before and after radiofrequency ablation. Their results revealed clear differences relying on whether HCC had a viral or nonviral etiology before radiofrequency ablation. Chen et al also applied ${ }^{1} \mathrm{H}-\mathrm{NMR}$-based metabolomics 
Table 3 Summary of recent metabolomic applications with respect to efficacy of anti-HCC agents and prognostic evaluation

\begin{tabular}{|c|c|c|c|c|}
\hline Reference & PubMed ID & Treatment & Sample & Method \\
\hline 81 & 21508352 & Belinostat and bortezomib & Hepl cells & NMR \\
\hline 82 & 26092946 & Sorafenib and everolimus & HepG2 cells & 2-D HRMAS 'H-NMR \\
\hline 84 & 26700591 & Geranylgeranic (GGA) acid & $\mathrm{HuH7}$ cells & UHPLC/TOF-MS \\
\hline 85 & 21843511 & Dehydroepiandrosterone & SK-HepI cells & RRLC-TOF-MS \\
\hline 86 & 28296595 & R- $\alpha$-lipoic acid (RLA) & H4IIEC 3 cells & GC-MS \\
\hline 87 & 24376596 & Acyclic retinoid (ACR) & $\mathrm{JHH7}$ cells & NMR and CE-TOF-MS \\
\hline 88 & 21327189 & Palmitate $(\mathrm{PA})$ and oleate $(\mathrm{OA})$ & H4IIEC3 cells & GC-MS \\
\hline 89 & 28884001 & $\begin{array}{l}\text { 6,7-dimethoxy-1,2,3,4-tetrahydro- } \\
\text { isoquinoline-3-carboxylic acid }\end{array}$ & Serum from DEN-induced hepatocarcinogenic rats & 'H-NMR \\
\hline 90 & 27077962 & Hispidulin & Urine samples from mice bearing $\mathrm{H} 22$ cells & UHPLC-QTOF-MS \\
\hline 91 & 27416811 & Physapubenolide (PB) & Plasma and liver tissue from mice bearing $\mathrm{H} 22$ cells & GC-MS \\
\hline 92 & $26744 I 70$ & Acyclic retinoid (ACR) & $\begin{array}{l}\text { Liver tissue samples from DEN-induced HCC } \\
\text { mouse model }\end{array}$ & $\begin{array}{l}\text { CE-TOF-MS and LC- } \\
\text { TOF-MS }\end{array}$ \\
\hline 93 & 28108381 & Shuihonghuazi (SHHZF) formula & Liver tissue from DEN-induced $\mathrm{HCC}$ rat model & HPLC/ESI-TOF-MS \\
\hline 94 & 24582150 & Surgical resection & $\begin{array}{l}\text { Plasma of } 18 \text { late-recurrent and } 22 \text { early-recurrent } \\
\text { HCC patients }\end{array}$ & LC-MS \\
\hline 95 & 22768978 & Surgical resection & $\begin{array}{l}\text { Urinary samples from } 19 \text { pairs of matched } \\
\text { preoperative and postoperative HCC patients and } \\
20 \text { healthy volunteers }\end{array}$ & GC-TOF-MS \\
\hline 96 & 27015127 & Radiofrequency ablation & $\begin{array}{l}\text { Serum samples from } 120 \mathrm{HCC} \text { patients before and } \\
\text { after radiofrequency ablation }\end{array}$ & 'H-NMR \\
\hline 97 & 27322079 & TACE or surgical treatments & $\begin{array}{l}\text { Plasma of } 57 \mathrm{HCC} \text { patients ( } 33 \text { underwent surgical } \\
\text { treatment, I } 8 \mathrm{TACE} \text { ), and } 60 \text { healthy control } \\
\text { subjects }\end{array}$ & 'H-NMR \\
\hline
\end{tabular}

Abbreviations: CE, capillary electrophoresis; DEN, diethylnitrosamine; DHEA, dehydroepiandrosterone; ESI, electrospray ionization; GC, gas chromatography HCC, hepatocellular carcinoma; HRMAS, high-resolution magic-angle spinning; MI, 6,7-dimethoxy-I,2,3,4-tetrahydro-isoquinoline-3-carboxylic acid; MS, mass spectrometry; MTHFR, methylenetetrahydrofolate reductase; NMR, nuclear magnetic resonance; PEMT, Phosphatidylethanolamine N-methyltransferase; PPP, pentose phosphate pathway; QTOF, quadrupole time of flight; RRLC, rapid-resolution LC; SAM, S-adenosylmethionine; TACE, transcatheter arterial chemoembolization; TCA, tricarboxylic acid; TOF, time of flight; UHPLC, ultrahigh-performance liquid chromatography.

to discriminate plasma metabolic profiles of HCC patients from different therapeutic backgrounds, such as transcatheter arterial chemoembolization or surgical treatment. They found that transcatheter arterial chemoembolization or surgical treatment did not immediately evoke apparent amelioration in the metabolic profiles of $\mathrm{HCC}$ patients. ${ }^{97}$ 


Significantly changed metabolites/pathways
Cotreatment induced increased amino acids and oxidative
stress
Addition of everolimus to sorafenib caused changes in
pyruvate, amino acid, methane, glyoxylate, and dicarboxylate
glycolysis/gluconeogenesis and glycerophospholipid and purine
metabolism
GGA increased fructose 6-phosphate and spermine while
decreasing fructose I,6-diphosphate and spermidine

DHEA treatment caused changes in metabolism of glutathione, lipid, SAM. and lysine

RLA treatment inhibited gluconeogenesis via suppressing ThrGly-Ser pathways, glycolysis, and lactic acid production ACR treatment suppressed ATP

Metabolite levels for TCA-cycle intermediates, PPP intermediates, and energy-storage metabolites changed between PA treatment alone and PA-OA cotreatment MI treatment modulated fatty acids, low-density lipoproteins, acetoacetate, choline, lysine, leucine, isoleucine, tyrosine, pyruvate, and creatine to normal levels

These changed pathways include pantothenate and CoA biosynthesis, glycine, serine, and threonine metabolism, nicotinate and nicotinamide metabolism, steroid-hormone biosynthesis; pyrimidine metabolism, and glyoxylate and dicarboxylate metabolism

PB disturbed metabolic pattern and significantly decreased lactate production

ACR significantly counteracted against acceleration of lipogenesis but not glucose metabolism in DEN-treated mouse liver

SHHZF inhibited abnormal metabolism of fatty-acid and bileacid metabolism

Metabolic differences found to be related to amino acid, bileacid, cholesterol, fatty-acid, phospholipid, and carbohydrate metabolism

Metabolism of TCA cycle, amino acids, nucleosides, and gut flora significantly changed after surgical resection

Impairment of glucose and lipid metabolism in liver cancer recurrence after curative treatment was found

HCC characterized by enhanced lipid metabolism and high consumption in response to liver injury
Main findings $\quad$ Validation

Cotreatment displayed synergistic antiproliferative property $\quad$ No

Sorafenib and everolimus have differential effects on HepG2 cells, and this phenomenon may explain (in part) the synergistic effects of sorafenib-everolimus combination therapy

GGA may shift HuH7 cells from aerobic glycolysis to mitochondrial respiration via upregulating TIGAR and SCO2 protein levels

The mitochondrial dysfunction caused by decreased SAM production and cardiolipin depletion underlie the antiproliferative effect of DHEA

This study may provide mechanistic insight into how RLA induces apoptosis in $\mathrm{HCC}$ cells

$A C R$ played its selective anti-HCC effect via suppressing the enhanced energy metabolism of HCC but not normal hepatic cells

Abnormal PPP fluxes and increased adenosine levels might contribute to differences between PA treatment alone and PAOA cotreatment

Ameliorations of these markers may be linked to repair of inflammation damage, improvement in energy metabolism, and reconstruction of cell-membrane injury

4-Phosphopantothenoylcysteine, glycine, niacinamide, cortisol, uracil, and 5-thymidylic acid are potential biomarkers that may explain the link between hispidulin and the metabolism of mice bearing neoplasms $(\mathrm{H} 22)$

PB induces apoptosis and decrease in glycolysis through the Akt-p53 pathway

Inhibition of linoleic acid metabolites, such as arachidonic acid, a proinflammatory precursor, played a crucial role in prevention by $A C R$

Anti-HCC property of SHHZF may be achieved via regulating the activities of lysophospholipase D, MTHFR, and PEMT Decreased levels of linolenic acid, docosahexaenoic acid, and polyunsaturated eicosapentaenoic acid the specific biomarker for early recurrence

Five metabolites (acotinic acid, phenylalanine, ethanolamine, ribose, and lactic acid) were identified as the biomarkers to predict early recurrence Statistical model showed significant differences depending on whether liver disease had a viral or nonviral etiology before radiofrequency ablation

TACE or surgical treatments did not immediately improve the metabolic profiles of $\mathrm{HCC}$ patients
No No

Yes

No

No

No

No

No

Yes

No

No

No

No

No

No

\section{Current challenges and future trends}

Altogether, though great achievements have been made in the field of HCC-biomarker discovery and therapy evaluation by metabolomics, this discipline is still in its infancy, and much exciting work remains to be done.

\section{Current challenges}

Although metabolomics has become a hot spot in the scientific community and better understanding of the complex pathophysiology of HCC has been achieved, this discipline still lags behind other-omics technologies to a great extent. ${ }^{98}$ The current challenges of metabolomics are primarily 
embodied in the following aspects. First, the most common challenge for metabolomics are its technical limitations. There are about 2,000 metabolites in living systems, and the number of metabolites detected by the current instrumentation is only in the hundreds. Although the development of analytical instrumentation has made much progress, most metabolites cannot be detected due to their dramatic concentration range and great complexity, and these undetectable metabolites may also play an important role in living systems. Second, as a high-throughput pattern-recognition method, metabolomics is based on the analysis of big data. Therefore, studies with small and inadequate samples would cause falsenegative results. Third, although most recent studies have offered us detailed information, such as demographic data for human subjects, there is still a lack of consistency on how the samples were selected and classified. For example, liver compensation and the treatment status of human subjects have often been inconsistently reported among different metabolomic studies on HCC. Fourth, a great challenge exists in data analysis of metabolomics, which is the most timeconsuming step of metabolomic studies. The limited database and analysis tools may lead to false-negative or false-positive results. Last but not least, a key challenge in metabolomics studies on HCC is bias among the researchers, which is an obstacle to bring metabolomics into clinical application. For example, very different biomarkers can be identified for the same cancer, due to different sample processing, different analytical platforms used, and different data processing.

\section{Future trends}

Further trends in metabolomic studies on HCC are as follows (Figure 2). In order to detect as many metabolites as possible, efforts should be made to advance the analysis platforms and computational methods in metabolomic studies. It is anticipated that future metabolomic studies will involve larger epidemiological studies that cover analysis of thousands of samples instead of the presently small sample sizes. ${ }^{99}$ A recently emerging trend in metabolomics is automation due to the need for quick and accurate quantification in the clinic that not only enlarges throughput but also elevates the reproducibility and reliability among different laboratories. ${ }^{100}$ Besides, standardization of metabolomic protocols, including sample collecting, handling, storing, techniques, hardware, and data processing, can also improve the reproducibility and reliability of metabolomics. ${ }^{101}$ A greatly recommended step of metabolomics is to validate metabolomic results. All the key metabolites obtained from metabolomic analysis should be validated to eliminate any unreliable findings by use of an additional set of samples. In the metabolomic studies on HCC we reviewed, only about $20 \%$ of publications had their findings validate. Therefore, it is a trend to validate key metabolites obtained by metabolomic analysis for both early HCC diagnosis and therapy evaluation. ${ }^{102}$ In order to gain a better processing and biological interpretation of metabolomic data, a more comprehensive database will be developed based on global body function, rather than organ-related function. It is widely accepted that a single analytical instrument can only identify a limited number of detectable metabolites. Therefore, an integration of different analytical instruments will be a trend to extend the number of detectable metabolites in biological samples and offer us more information. ${ }^{103}$ Most of the recent metabolomic studies on HCC have been conducted only at the metabolic level and lacked integrated information on other levels, such as DNA, RNA, and proteins. Since no

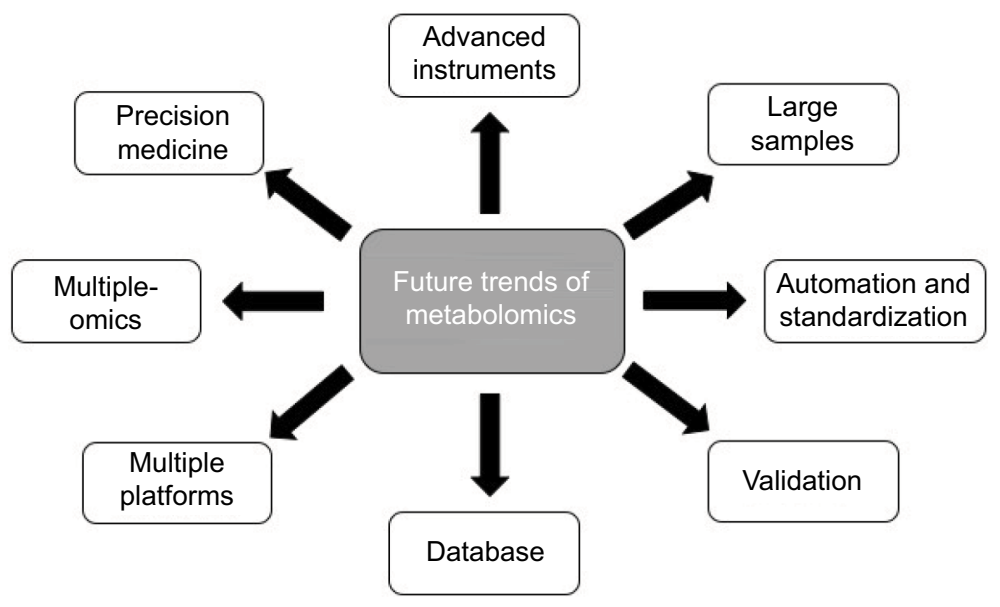

Figure 2 Future trends in metabolomics. 
single technology can provide us the entire spectrum of the HCC phenotype, integration of metabolomic results with markers obtained from the upstream -omics technologies is a new trend to increase our understanding of the pathological progression of HCC, as well as responses to therapy. ${ }^{104}$ The concept of "precision medicine" (also called personalized medicine) in cancer health care is gradually gaining interest, of which the goal is to use advanced technologies to customize an individual's medical treatment based on their own biomarker profiles. Given that metabolomics is relatively inexpensive and provides valuable biomarkers in disease diagnosis and therapy monitoring, there can be little doubt that it will be useful in precision medicine to guide clinical strategies from personal disease diagnosis and monitoring to selecting a suitable therapy and finally to following patient prognosis in the future. ${ }^{105}$ Although the consideration of these challenges and future trends will not be satisfied at present or in the near future, it is essential that they are highly recognized and being discussed on a larger scale.

\section{Conclusion}

In summary, metabolomics is a burgeoning science with a combination of analytical chemistry, biochemistry, bioinformatics, and medicine, which is well suited to studies of HCC. It can be applied not only to identify the sensitive and specific biomarkers of HCC in a noninvasive and nondestructive way at the early stage but also to evaluate the efficacy of treatment and prognosis. Valuable information regarding HCC diagnosis, prognosis, and therapy can be obtained from these metabolomic studies of HCC. Collectively, metabolomics provides us new insights into the diagnosis, prognosis, and therapeutic evaluation of HCC. With the coming era of precision medicine, metabolomics will undoubtedly play an active role in selecting a suitable therapy tailored to an individual patient in the future. Although technological advances have brought metabolomics into the spotlight, metabolomics is still in its infancy, and much exciting work remains to be done.

\section{Acknowledgments}

The study was financially supported by grants from the research council of the University of Hong Kong (project codes 104004092, 104003919, 104004460, 104004745, and 104004746), the Research Grants Committee (RGC) of Hong Kong, HKSAR (project codes 766211, 17152116), Wong's Donation for Modern Oncology of Chinese Medicine (project code 200006276), and the Shenzhen Basic Research Program (project code JCYJ20140903112959964).

\section{Disclosure}

The authors report no conflicts of interest in this work.

\section{References}

1. Patel M, Shariff MI, Ladep NG, et al. Hepatocellular carcinoma: diagnostics and screening. J Eval Clin Pract. 2012;18(2):335-342.

2. Ohata K, Hamasaki K, Toriyama K, Ishikawa H, Nakao K, Eguchi K. High viral load is a risk factor for hepatocellular carcinoma in patients with chronic hepatitis B virus infection. J Gastroenterol Hepatol. 2004;19(6):670-675.

3. Bosch FX, Ribes J, Diaz M, Cleries R. Primary liver cancer: worldwide incidence and trends. Gastroenterology. 2004;127(5 Suppl 1):S5-S16.

4. Morgan TR, Mandayam S, Jamal MM. Alcohol and hepatocellular carcinoma. Gastroenterology. 2004;127(5 Suppl 1):S87-S96.

5. Tsai JF, Chuang LY, Jeng JE, et al. Betel quid chewing as a risk factor for hepatocellular carcinoma: a case-control study. Br J Cancer. 2001;84(5):709-713.

6. Groopman JD, Scholl P, Wang JS. Epidemiology of human aflatoxin exposures and their relationship to liver cancer. Prog Clin Biol Res. 1996;395:211-222.

7. Regimbeau JM, Colombat M, Mognol P, et al. Obesity and diabetes as a risk factor for hepatocellular carcinoma. Liver Transpl. 2004;10(2 Suppl 1):S69-S73.

8. Justesen P, Fenger C, Høilund-Carlsen PF, et al. Hepatocellulært karcinom og andre levertumorer [Hepatocellular carcinoma and other liver tumors]. Ugeskr Laeger. 2006;168(19):1876. Danish.

9. Taketa K. $\alpha$-Fetoprotein: reevaluation in hepatology. Hepatology. 1990;12(6):1420-1432.

10. Wishart DS, Tzur D, Knox C, et al. HMDB: the Human Metabolome Database. Nucleic Acids Res. 2007;35(Database):D521-D526.

11. Ganti S, Weiss RH. Urine metabolomics for kidney cancer detection and biomarker discovery. Urol Oncol. 2011;29(5):551-557.

12. Zhang A, Sun H, Wang P, Han Y, Wang X. Recent and potential developments of biofluid analyses in metabolomics. J Proteomics. 2012;75(4):1079-1088.

13. Catchpole GS, Beckmann M, Enot DP, et al. Hierarchical metabolomics demonstrates substantial compositional similarity between genetically modified and conventional potato crops. Proc Natl Acad Sci U S A. 2005;102(40):14458-14462.

14. Armitage EG, Southam AD. Monitoring cancer prognosis, diagnosis and treatment efficacy using metabolomics and lipidomics. Metabolomics. 2016;12:146.

15. Kaddurah-Daouk R, Weinshilboum R. Metabolomic signatures for drug response phenotypes: pharmacometabolomics enables precision medicine. Clin Pharmacol Ther. 2015;98(1):71-75.

16. Liu C, Alessandro A, Xia Y. Metabolomic approach in probing drug candidates. Curr Top Med Chem. 2017;17(15):1741-1749.

17. Ward PS, Thompson CB. Metabolic reprogramming: a cancer hallmark even Warburg did not anticipate. Cancer Cell. 2012;21(3):297-308.

18. Serkova NJ, Spratlin JL, Eckhardt SG. NMR-based metabolomics: translational application and treatment of cancer. Curr Opin Mol Ther. 2007;9(6):572-585.

19. Wang X, Zhang A, Sun H. Power of metabolomics in diagnosis and biomarker discovery of hepatocellular carcinoma. Hepatology. 2013;57(5):2072-2077.

20. Chaiteerakij R, Addissie BD, Roberts LR. Update on biomarkers of hepatocellular carcinoma. Clin Gastroenterol Hepatol. 2015;13(2): 237-245.

21. Kimhofer T, Fye H, Taylor-Robinson S, Thursz M, Holmes E. Proteomic and metabonomic biomarkers for hepatocellular carcinoma: a comprehensive review. Br J Cancer. 2015;112(7):1141-1156.

22. Fitian AI, Cabrera R. Disease monitoring of hepatocellular carcinoma through metabolomics. World J Hepatol. 2017;9(1):1-17.

23. Tong A, Wu L, Lin Q, et al. Proteomic analysis of cellular protein alterations using a hepatitis B virus-producing cellular model. Proteomics. 2008;8(10):2012-2023. 
24. Yang F, Yan S, He Y, et al. Expression of hepatitis B virus proteins in transgenic mice alters lipid metabolism and induces oxidative stress in the liver. J Hepatol. 2008;48(1):12-19.

25. Li H, Zhu W, Zhang L, et al. The metabolic responses to hepatitis B virus infection shed new light on pathogenesis and targets for treatment. Sci Rep. 2015;5:8421.

26. Na TY, Shin YK, Roh KJ, et al. Liver X receptor mediates hepatitis $\mathrm{B}$ virus $\mathrm{X}$ protein-induced lipogenesis in hepatitis $\mathrm{B}$ virus-associated hepatocellular carcinoma. Hepatology. 2009;49(4):1122-1131.

27. Yue D, Zhang YW, Cheng LL, et al. Hepatitis B virus $X$ protein (HBx)-induced abnormalities of nucleic acid metabolism revealed by ${ }^{1}$ H-NMR-based metabonomics. Sci Rep. 2016;6:24430.

28. Dai Y, Cros MP, Pontoizeau C, Elena-Hermann B, Bonn GK, Hainaut P. Downregulation of transcription factor $\mathrm{E} 4 \mathrm{~F} 1$ in hepatocarcinoma cells: HBV-dependent effects on autophagy, proliferation and metabolism. Carcinogenesis. 2014;35(3):635-650.

29. Xie Q, Fan F, Wei W, et al. Multi-omics analyses reveal metabolic alterations regulated by hepatitis $\mathrm{B}$ virus core protein in hepatocellular carcinoma cells. Sci Rep. 2017;7:41089.

30. Min HK, Sookoian S, Pirola CJ, Cheng J, Mirshahi F, Sanyal AJ. Metabolic profiling reveals that PNPLA3 induces widespread effects on metabolism beyond triacylglycerol remodeling in Huh-7 hepatoma cells. Am J Physiol Gastrointest Liver Physiol. 2014;307(1): G66-G76.

31. Fang M, Dewaele S, Zhao YP, et al. Serum N-glycome biomarker for monitoring development of DENA-induced hepatocellular carcinoma in rat. Mol Cancer. 2010;9:215.

32. Hong EJ, Levasseur MP, Dufour CR, Perry MC, Giguere V. Loss of estrogen-related receptor $\alpha$ promotes hepatocarcinogenesis development via metabolic and inflammatory disturbances. Proc Natl Acad Sci U S A. 2013;110(44):17975-17980.

33. Tan Y, Yin P, Tang L, et al. Metabolomics study of stepwise hepatocarcinogenesis from the model rats to patients: potential biomarkers effective for small hepatocellular carcinoma diagnosis. Mol Cell Proteomics. 2012;11(2):M111.010694.

34. Zeng J, Huang X, Zhou L, et al. Metabolomics identifies biomarker pattern for early diagnosis of hepatocellular carcinoma: from diethylnitrosamine treated rats to patients. Sci Rep. 2015;5:16101.

35. Huang X, Zeng J, Zhou L, Hu C, Yin P, Lin X. A new strategy for analyzing time-series data using dynamic networks: identifying prospective biomarkers of hepatocellular carcinoma. Sci Rep. 2016;6:32448.

36. Li ZF, Wang J, Huang C, et al. Gas chromatography/time-of-flight mass spectrometry-based metabonomics of hepatocarcinoma in rats with lung metastasis: elucidation of the metabolic characteristics of hepatocarcinoma at formation and metastasis. Rapid Commun Mass Spectrom. 2010;24(18):2765-2775.

37. Wang J, Zhang S, Li Z, et al. 'H-NMR-based metabolomics of tumor tissue for the metabolic characterization of rat hepatocellular carcinoma formation and metastasis. Tumour Biol. 2011;32(1):223-231.

38. Teng CF, Hsieh WC, Yang CW, et al. A biphasic response pattern of lipid metabolomics in the stage progression of hepatitis $\mathrm{B}$ virus $\mathrm{X}$ tumorigenesis. Mol Carcinog. 2016;55(1):105-114.

39. Fan T, Rong Z, Dong J, et al. Metabolomic and transcriptomic profiling of hepatocellular carcinomas in Hras $12 \mathrm{~V}$ transgenic mice. Cancer Med. 2017;6(10):2370-2384.

40. Li S, Liu H, Jin Y, Lin S, Cai Z, Jiang Y. Metabolomics study of alcohol-induced liver injury and hepatocellular carcinoma xenografts in mice. J Chromatogr B Analyt Technol Biomed Life Sci. 2011;879(24):2369-2375.

41. Assi N, Fages A, Vineis P, et al. A statistical framework to model the meeting-in-the-middle principle using metabolomic data: application to hepatocellular carcinoma in the EPIC study. Mutagenesis. 2015;30(6):743-753

42. Fages A, Duarte-Salles T, Stepien M, et al. Metabolomic profiles of hepatocellular carcinoma in a European prospective cohort. BMC Med. 2015;13:242.
43. Chen F, Xue J, Zhou L, Wu S, Chen Z. Identification of serum biomarkers of hepatocarcinoma through liquid chromatography/mass spectrometry-based metabonomic method. Anal Bioanal Chem. 2011;401(6):1899-1904.

44. Chen S, Kong H, Lu X, et al. Pseudotargeted metabolomics method and its application in serum biomarker discovery for hepatocellular carcinoma based on ultra high-performance liquid chromatography/triple quadrupole mass spectrometry. Anal Chem. 2013;85(17):8326-8333.

45. Zeng Z, Liu X, Dai W, et al. Ion fusion of high-resolution LC-MSbased metabolomics data to discover more reliable biomarkers. Anal Chem. 2014;86(8):3793-3800.

46. Liang Q, Liu H, Wang C, Li B. Phenotypic characterization analysis of human hepatocarcinoma by urine metabolomics approach. Sci Rep. 2016;6:19763.

47. Zhang A, Sun H, Yan G, Han Y, Ye Y, Wang X. Urinary metabolic profiling identifies a key role for glycocholic acid in human liver cancer by ultra-performance liquid-chromatography coupled with high-definition mass spectrometry. Clin Chim Acta. 2013;418:86-90.

48. Chen T, Xie G, Wang X, et al. Serum and urine metabolite profiling reveals potential biomarkers of human hepatocellular carcinoma. $\mathrm{Mol}$ Cell Proteomics. 2011;10(7):M110.004945.

49. Nahon P, Amathieu R, Triba MN, et al. Identification of serum proton NMR metabolomic fingerprints associated with hepatocellular carcinoma in patients with alcoholic cirrhosis. Clin Cancer Res. 2012;18(24):6714-6722.

50. Ressom HW, Xiao JF, Tuli L, et al. Utilization of metabolomics to identify serum biomarkers for hepatocellular carcinoma in patients with liver cirrhosis. Anal Chim Acta. 2012;743:90-100.

51. Xiao JF, Varghese RS, Zhou B, et al. LC-MS based serum metabolomics for identification of hepatocellular carcinoma biomarkers in Egyptian cohort. J Proteome Res. 2012;11(12):5914-5923.

52. Liu Y, Hong Z, Tan G, et al. NMR and LC-MS-based global metabolomics to identify serum biomarkers differentiating hepatocellular carcinoma from liver cirrhosis. Int J Cancer. 2014;135(3):658-668.

53. Zeng J, Yin P, Tan Y, et al. Metabolomics study of hepatocellular carcinoma: discovery and validation of serum potential biomarkers by using capillary electrophoresis-mass spectrometry. J Proteome Res. 2014;13(7):3420-3431.

54. Fitian AI, Nelson DR, Liu C, Xu Y, Ararat M, Cabrera R. Integrated metabolomic profiling of hepatocellular carcinoma in hepatitis C cirrhosis through GC-MS and UPLC-MS-MS. Liver Int. 2014;34(9):1428-1444.

55. Ranjbar MR, Luo Y, Di Poto C, et al. GC-MS based plasma metabolomics for identification of candidate biomarkers for hepatocellular carcinoma in Egyptian cohort. PLoS One. 2015;10(6):e0127299.

56. Di Poto C, Ferrarini A, Zhao Y, et al. Metabolomic characterization of hepatocellular carcinoma in patients with liver cirrhosis for biomarker discovery. Cancer Epidemiol Biomarkers Prev. 2017;26(5):675-683.

57. Sanabria JR, Kombu RS, Zhang GF, et al. Glutathione species and metabolomic prints in subjects with liver disease as biological markers for the detection of hepatocellular carcinoma. HPB (Oxford). 2016;18(12):979-990.

58. Wang B, Chen D, Chen Y, et al. Metabonomic profiles discriminate hepatocellular carcinoma from liver cirrhosis by ultraperformance liquid chromatography-mass spectrometry. JProteome Res. 2012;11(2):1217-1227.

59. Shao Y, Zhu B, Zheng R, et al. Development of urinary pseudotargeted LC-MS-based metabolomics method and its application in hepatocellular carcinoma biomarker discovery. J Proteome Res. 2015;14(2):906-916.

60. Shariff MI, Gomaa AI, Cox IJ, et al. Urinary metabolic biomarkers of hepatocellular carcinoma in an Egyptian population: a validation study. J Proteome Res. 2011;10(4):1828-1836.

61. Osman D, Ali O, Obada M, El-Mezayen H, El-Said H. Chromatographic determination of some biomarkers of liver cirrhosis and hepatocellular carcinoma in Egyptian patients. Biomed Chromatogr. 2017;31(6):e3893. 
62. Dai W, Yin P, Chen P, et al. Study of urinary steroid hormone disorders: difference between hepatocellular carcinoma in early stage and cirrhosis. Anal Bioanal Chem. 2014;406(18):4325-4335.

63. Cao H, Huang $\mathrm{H}, \mathrm{Xu} \mathrm{W}$, et al. Fecal metabolome profiling of liver cirrhosis and hepatocellular carcinoma patients by ultra performance liquid chromatography-mass spectrometry. Anal Chim Acta. 2011;691(1-2):68-75.

64. Patterson AD, Maurhofer O, Beyoglu D, et al. Aberrant lipid metabolism in hepatocellular carcinoma revealed by plasma metabolomics and lipid profiling. Cancer Res. 2011;71(21):6590-6600.

65. Zhou L, Ding L, Yin P, et al. Serum metabolic profiling study of hepatocellular carcinoma infected with hepatitis $\mathrm{B}$ or hepatitis $\mathrm{C}$ virus by using liquid chromatography-mass spectrometry. J Proteome Res. 2012;11(11):5433-5442.

66. Tian S, Chang HH, Wang C, Jiang J, Wang X, Niu J. Multi-TGDR, a multi-class regularization method, identifies the metabolic profiles of hepatocellular carcinoma and cirrhosis infected with hepatitis B or hepatitis C virus. BMC Bioinformatics. 2014;15:97.

67. Bowers J, Hughes E, Skill N, Maluccio M, Raftery D. Detection of hepatocellular carcinoma in hepatitis $\mathrm{C}$ patients: biomarker discovery by LC-MS. J Chromatogr B Analyt Technol Biomed Life Sci. 2014;966:154-162.

68. Wei S, Suryani Y, Gowda GA, Skill N, Maluccio M, Raftery D. Differentiating hepatocellular carcinoma from hepatitis $\mathrm{C}$ using metabolite profiling. Metabolites. 2012;2(4):701-716.

69. Baniasadi H, Gowda GA, Gu H, et al. Targeted metabolic profiling of hepatocellular carcinoma and hepatitis C using LC-MS/MS. Electrophoresis. 2013;34(19):2910-2917.

70. Gao R, Cheng J, Fan C, et al. Serum metabolomics to identify the liver disease-specific biomarkers for the progression of hepatitis to hepatocellular carcinoma. Sci Rep. 2015;5:18175.

71. Gong ZG, Zhao W, Zhang J, et al. Metabolomics and eicosanoid analysis identified serum biomarkers for distinguishing hepatocellular carcinoma from hepatitis B virus-related cirrhosis. Oncotarget 2017;8(38):63890-63900.

72. Lin X, Gao J, Zhou L, Yin P, Xu G. A modified k-TSP algorithm and its application in LC-MS-based metabolomics study of hepatocellular carcinoma and chronic liver diseases. J Chromatogr B Analyt Technol Biomed Life Sci. 2014;966:100-108.

73. Zhou L, Wang Q, Yin P, et al. Serum metabolomics reveals the deregulation of fatty acids metabolism in hepatocellular carcinoma and chronic liver diseases. Anal Bioanal Chem. 2012;403(1):203-213.

74. Soga T, Sugimoto M, Honma M, et al. Serum metabolomics reveals $\gamma$-glutamyl dipeptides as biomarkers for discrimination among different forms of liver disease. J Hepatol. 2011;55(4):896-905.

75. Huang Q, Tan Y, Yin P, et al. Metabolic characterization of hepatocellular carcinoma using nontargeted tissue metabolomics. Cancer Res. 2013;73(16):4992-5002.

76. Liu SY, Zhang RL, Kang H, Fan ZJ, Du Z. Human liver tissue metabolic profiling research on hepatitis B virus-related hepatocellular carcinoma. World J Gastroenterol. 2013;19(22):3423-3432.

77. Budhu A, Roessler S, Zhao X, et al. Integrated metabolite and gene expression profiles identify lipid biomarkers associated with progression of hepatocellular carcinoma and patient outcomes. Gastroenterology. 2013;144(5):1066-1075 e1061.

78. Beyoğlu D, Imbeaud S, Maurhofer O, et al. Tissue metabolomics of hepatocellular carcinoma: tumor energy metabolism and the role of transcriptomic classification. Hepatology. 2013;58(1):229-238.

79. Murakami Y, Kubo S, Tamori A, et al. Comprehensive analysis of transcriptome and metabolome analysis in intrahepatic cholangiocarcinoma and hepatocellular carcinoma. Sci Rep. 2015;5: 16294.

80. Darpolor MM, Basu SS, Worth A, et al. The aspartate metabolism pathway is differentiable in human hepatocellular carcinoma: transcriptomics and ${ }^{13} \mathrm{C}$-isotope based metabolomics. NMR Biomed. 2014;27(4):381-389.
81. Spratlin JL, Pitts TM, Kulikowski GN, et al. Synergistic activity of histone deacetylase and proteasome inhibition against pancreatic and hepatocellular cancer cell lines. Anticancer Res. 2011;31(4):1093-1103.

82. Zheng JF, Lu J, Wang XZ, Guo WH, Zhang JX. Comparative metabolomic profiling of hepatocellular carcinoma cells treated with sorafenib monotherapy vs. sorafenib-everolimus combination therapy. $\mathrm{Med} \mathrm{Sci}$ Monit. 2015;21:1781-1791.

83. Asghar U, Meyer T. Are there opportunities for chemotherapy in the treatment of hepatocellular cancer? J Hepatol. 2012;56(3):686-695.

84. Iwao C, Shidoji Y. Upregulation of energy metabolism-related, p53-target TIGAR and SCO2 in HuH-7 cells with p53 mutation by geranylgeranoic acid treatment. Biomed Res. 2015;36(6):371-381.

85. Cheng ML, Shiao MS, Chiu DT, Weng SF, Tang HY, Ho HY. Biochemical disorders associated with antiproliferative effect of dehydroepiandrosterone in hepatoma cells as revealed by LC-based metabolomics. Biochem Pharmacol. 2011;82(11):1549-1561.

86. Ikuta N, Chikamoto K, Asano Y, et al. Time course effect of R- $\alpha$-lipoic acid on cellular metabolomics in cultured hepatoma cells. J Med Food. 2017;20(3):211-222.

87. Qin XY, Wei F, Tanokura M, et al. The effect of acyclic retinoid on the metabolomic profiles of hepatocytes and hepatocellular carcinoma cells. PLoS One. 2013;8(12):e82860.

88. Noguchi Y, Young JD, Aleman JO, Hansen ME, Kelleher JK, Stephanopoulos G. Tracking cellular metabolomics in lipoapoptosis- and steatosis-developing liver cells. Mol Biosyst. 2011;7(5):1409-1419.

89. Kumar P, Singh AK, Raj V, et al. 6,7-Dimethoxy-1,2,3,4-tetrahydroisoquinoline-3-carboxylic acid attenuates hepatocellular carcinoma in rats with NMR-based metabolic perturbations. Future Sci OA. 2017;3(3):FSO202.

90. Li F, Li X, Miao Y, et al. UHPLC-MS-based metabolomics analysis on mice bearing neoplasm (H22) for hispidulin. J Pharm Biomed Anal. 2016;125:310-318.

91. Ma T, Fan BY, Zhang C, et al. Metabonomics applied in exploring the antitumour mechanism of physapubenolide on hepatocellular carcinoma cells by targeting glycolysis through the Akt-p53 pathway. Sci Rep. 2016;6:29926.

92. Qin XY, Tatsukawa H, Hitomi K, et al. Metabolome analyses uncovered a novel inhibitory effect of acyclic retinoid on aberrant lipogenesis in a mouse diethylnitrosamine-induced hepatic tumorigenesis model. Cancer Prev Res (Phila). 2016;9(3):205-214.

93. Bao Y, Wang S, Yang X, Li T, Xia Y, Meng X. Metabolomic study of the intervention effects of shuihonghuazi formula, a traditional Chinese medicinal formulae, on hepatocellular carcinoma (HCC) rats using performance HPLC/ESI-TOF-MS. J Ethnopharmacol. 2017;198:468-478.

94. Zhou L, Liao Y, Yin P, et al. Metabolic profiling study of early and late recurrence of hepatocellular carcinoma based on liquid chromatography-mass spectrometry. J Chromatogr B Analyt Technol Biomed Life Sci. 2014;966:163-170.

95. Ye G, Zhu B, Yao Z, et al. Analysis of urinary metabolic signatures of early hepatocellular carcinoma recurrence after surgical removal using gas chromatography-mass spectrometry. J Proteome Res. 2012;11(8):4361-4372.

96. Goossens C, Nahon P, le Moyec L, et al. Sequential serum metabolomic profiling after radiofrequency ablation of hepatocellular carcinoma reveals different response patterns according to etiology. J Proteome Res. 2016;15(5):1446-1454.

97. Chen Y, Zhou J, Li J, Feng J, Chen Z, Wang X. Plasma metabolomic analysis of human hepatocellular carcinoma: diagnostic and therapeutic study. Oncotarget. 2016;7(30):47332-47342.

98. Spratlin JL, Serkova NJ, Eckhardt SG. Clinical applications of metabolomics in oncology: a review. Clin Cancer Res. 2009;15(2):431-440.

99. Gika HG, Theodoridis GA, Plumb RS, Wilson ID. Current practice of liquid chromatography-mass spectrometry in metabolomics and metabonomics. J Pharm Biomed Anal. 2014;87:12-25. 
100. Grebe SK, Singh RJ. LC-MS/MS in the clinical laboratory: where to from here? Clin Biochem Rev. 2011;32(1):5-31.

101. Vermeersch KA, Styczynski MP. Applications of metabolomics in cancer research. J Carcinog. 2013;12:9.

102. Hwang VJ, Weiss RH. Metabolomic profiling for early cancer detection: current status and future prospects. Expert Opin Drug Metab Toxicol. Epub 2016 Sep 13.
103. Goodacre R, Vaidyanathan S, Dunn WB, Harrigan GG, Kell DB. Metabolomics by numbers: acquiring and understanding global metabolite data. Trends Biotechnol. 2004;22(5):245-252.

104. Nicholson JK, Lindon JC. Systems biology: metabonomics. Nature. 2008;455(7216):1054-1056.

105. Everett JR. Pharmacometabonomics in humans: a new tool for personalized medicine. Pharmacogenomics. 2015;16(7):737-754.

\section{Publish your work in this journal}

Cancer Management and Research is an international, peer-reviewed open access journal focusing on cancer research and the optimal use of preventative and integrated treatment interventions to achieve improved outcomes, enhanced survival and quality of life for the cancer patient. The manuscript management system is completely online and includes a very quick and fair peer-review system, which is all easy to use. Visit http://www.dovepress.com/testimonials.php to read real quotes from published authors. 